Research Article

\title{
Improved Artificial Potential Field Method Applied for AUV Path Planning
}

\author{
Xiaojing Fan $\mathbb{D},{ }^{1,2}$ Yinjing Guo $\mathbb{D},{ }^{1,3}$ Hui Liu $\mathbb{D},{ }^{1}$ Bowen Wei $\mathbb{D},{ }^{4}$ and Wenhong Lyu $\mathbb{D}^{5}$ \\ ${ }^{1}$ College of Electronic and Information Engineering, Shandong University of Science and Technology, Qingdao 266590, China \\ ${ }^{2}$ School of Computer Engineering, Weifang University, Weifang 261061, China \\ ${ }^{3}$ Qingdao Intelligence Ocean Technology Co., Ltd., Qingdao 266590, China \\ ${ }^{4}$ Shandong Branch of China Mobile Communication Group Co., Ltd., Binzhou 256600, China \\ ${ }^{5}$ College of Transportation, Shandong University of Science and Technology, Qingdao 266590, China \\ Correspondence should be addressed to Yinjing Guo; gyj@sdust.edu.cn
}

Received 22 January 2020; Accepted 9 March 2020; Published 27 April 2020

Academic Editor: Krzysztof Puszynski

Copyright (C) 2020 Xiaojing Fan et al. This is an open access article distributed under the Creative Commons Attribution License, which permits unrestricted use, distribution, and reproduction in any medium, provided the original work is properly cited.

\begin{abstract}
With the topics related to the intelligent AUV, control and navigation have become one of the key researching fields. This paper presents a concise and reliable path planning method for AUV based on the improved APF method. AUV can make the decision on obstacle avoidance in terms of the state of itself and the motion of obstacles. The artificial potential field (APF) method has been widely applied in static real-time path planning. In this study, we present the improved APF method to solve some inherent shortcomings, such as the local minima and the inaccessibility of the target. A distance correction factor is added to the repulsive potential field function to solve the GNRON problem. The regular hexagon-guided method is proposed to improve the local minima problem. Meanwhile, the relative velocity method about the moving objects detection and avoidance is proposed for the dynamic environment. This method considers not only the spatial location but also the magnitude and direction of the velocity of the moving objects, which can avoid dynamic obstacles in time. So the proposed path planning method is suitable for both static and dynamic environments. The virtual environment has been built, and the emulation has been in progress in MATLAB. Simulation results show that the proposed method has promising feasibility and efficiency in the AUV real-time path planning. We demonstrate the performance of the proposed method in the real environment. Experimental results show that the proposed method is capable of avoiding the obstacles efficiently and finding an optimized path.
\end{abstract}

\section{Introduction}

Autonomous underwater vehicle (AUV) refers to an untethered underwater vehicle that can sail underwater by remote control or autonomous control [1]. AUV is an essential tool for human beings to expand cognition and explore the world under the sea. It can operate autonomously in the place where human beings cannot reach [2] and has a very profound value of research [3-7] and application [8]. The circumstances of underwater are not set in stone. When cruising in the sea, AUV will come across static as well as dynamic surroundings, which means the obstacles may be stationary or moving or both, for example, fish, moving obstacles (such as other AUV) and unknown static obstacles (such as reefs). The AUV must be able to find an efficient and safe path to avoid collision with them. So the intelligent path planning is considered to be a key technology for an AUV's utility and mission success $[9,10]$.

Path planning refers to the fact that the robot finds a collision-free optimal path from the start point to the goal point in the workspace with obstacles, on the premise of the one or more optimization criteria among the shortest moving path, the shortest moving time, and the minimum working cost [11-13]. Numerous algorithms on robot path planning have been developed. The primary navigation algorithms can be broadly classified into two main approaches: classical algorithms and heuristics algorithms [14]. The classical approaches include roadmap building, cell 
decomposition, reactive approaches, and artificial potential field (APF) method. Roadmap building algorithms are a group of computational geometry-based approaches to path planning [15], which included visibility graph [16], Voronoi diagram [15], subgoal network [17], and silhouette approach [18]. These algorithms extend the obstacles in the workspace into polygons. The connection is established among the corner point of polygonal obstacles, the start point, and the goal point to achieve the shortest path search. The algorithm of cell decomposition is proposed by Cai and Ferrari [19]. Its principle is to decompose the environment of the robot into a set of nonoverlapping cells. The cells are used to construct a connectivity graph. The graph would be transformed into a decision tree from which an optimal sensing strategy can be computed to perform the path planning. Reactive approaches, namely, fuzzy logic [20], neural networks [21], and neurofuzzy systems [22], are also the algorithms that have served as the core methodology to develop path planning. It simulates the workspace of the robot as a network of neurons. Use the information received from the sensors, such as the obstacle position and the motion state as inputs of the neural network. Take the robot movement direction and the steering angle as outputs through iterative calculations. The robot can avoid the obstacles and move toward the goal point. The APF method [23] is the virtual force method that was first proposed by Khatib in 1986. The movement of the robot in the environmental space is transformed into the virtual force field motion. The attractive force of the goal point attracts the robot to move toward it. The repulsive force of the obstacles prevents the robot from moving toward it. The resultant force is the combination of the attractive force of the goal point and the repulsive forces of all obstacles. The magnitude and direction of the resultant force determine the motion state of the robot. Heuristics algorithms [24] contain $A *$ algorithm [25], Dijkstra algorithm [26], BFS algorithm [27], Bio-inspired algorithms [28-33], and so on [34,35]. These algorithms can yield good solutions, but not necessarily the optimum. All the mentioned algorithms have their respective advantages and disadvantages. They are not mutually independent but deeply correlated to each other. In many applications, some of them can be combined to derive the most effective path planning manner [14].

AUV needs to work across a broad range of marine environments. It has higher requirements for real-time computation and energy consumption of the path planning method. The APF method is the most widely used one in AUV path planning because the model is simple and applicable for real-time implementation [36]. When AUV detects an unknown obstacle, it takes the current position as the start point and the next turning point in the global path planning as the goal point. Construct an attractive potential field at the goal point and the repulsive potential field around the obstacle. AUV moves toward the goal point in the joint action of the attractive force and the repulsive forces [10]. However, there are some inherent problems in the traditional APF method: (1) goal nonreachable with obstacles nearby (GNRON), (2) the local minima problem, and (3) dynamic environment obstacle avoidance. Many proposals have emerged [37-42] to overcome such drawbacks. Matoui et al. [37] handle the local minimum problem using the nonminimum speed algorithm. Weerakoon et al. [38] overcome the local minima problem by replacing traditionally used functions with exponential functions. It generates a new repulsive force to the primary force when the robot detects an obstacle within its sensory range. Sun et al. [39] use a dynamic window approach to solve the local minimum problem. It employs a cost function to evaluate simulated trajectories, such that the local minimum region can be found in the prior step. Liang et al. [40] propose the approach of sector division to increase the virtual obstacle in the appropriate range around the local minimum point. Under the combined action of the original obstacles and the target point, the force to the mobile robot is generated. Yang et al. [41] build a new repulsive potential function that takes the relative distance between the robot and the goal into consideration to overcome the GNRON problem. It uses the potential filling method that sets an additional potential field in the area to pull the robot away from the local minima. $\mathrm{Li}$ et al. [42] employ virtual local target and repulsive force disappearance method to eliminate local minimum caused by traditional APF when attractive force and repulsive force are in collinear but opposite direction.

For the dynamic environment, Sun et al. [39] define an evolutionary potential field function based on an improved minimum danger index. It can plan an optimal path using not only the relative distance and velocity direction information, but also the magnitude information of the robot and obstacle speeds. Ge and Cui [43] propose a new potential field method in a dynamic environment where both the target and obstacles are moving. The new potential function takes into account not only the position but also the velocity of the robot (relative to target and obstacles). The virtual force is defined as the negative gradient of the potential with respect to position and velocity. The total virtual force then determines the motion of the mobile robot. Montiel et al. [44] introduce a parallel evolutionary artificial potential field for the dynamic environment. It can achieve controllability in complex real-world sceneries with dynamic obstacles if a reachable configuration set exists. Zhu et al. [45] take account of both the ocean current and moving obstacles influence underwater. It proposes an integrated AUV path planning algorithm through a combination of velocity synthesis (VS) and an enhanced APF algorithm. Cheng et al. [46] propose a novel integrated AUV path planning algorithm by combining the velocity synthesis and artificial potential field algorithm. The improved artificial potential field method is used to avoid dynamic obstacles effectively, and the velocity synthesis algorithm is used to achieve the optimal path. These algorithms may help the robot escaping from inherent problems and make intelligent path planning. Based on previous studies and the basic theory of the APF method, there is still some room for improvement to achieve more simple and efficient obstacle avoidance path planning.

In this paper, we present a real-time path planning method for the AUV based on the improved APF method. The main contributions of this paper can be summarized as follows. First, the modified APF method is developed to 
address the inherent problems: a distance correction factor is added to the repulsive potential field function to solve the GNRON problem; the regular hexagon-guided (RHG) method is combined with the traditional APF method to address the local minima problem. If the AUV trapped in a local minimum region, the system can construct a virtual regular hexagon helping it get out of the difficult position. The distance of a one-step position is taken as the side length of the regular hexagon. Second, the relative velocity method, an evolutionary potential field method, is presented to adapt the dynamic environment obstacle avoidance. This method considers not only the spatial location but also the magnitude and direction of the velocity of the moving obstacles. Third, the proposed path planning method can select a suitable obstacle avoidance strategy by apperceiving the motion of the obstacles (static or moving) and the running status of the AUV (such as whether trapped in a local minimum region). So the proposed method can not only reduce the burden of computation but also react to sensory data as quickly as possible for the static as well as dynamic environments. AUV can make the decision to avoid both static and moving obstacles. We demonstrate the proposed method in the simulation and real environments, respectively, which shows the effectiveness of our method.

This paper is organized as follows. In Section 2, we briefly review the mathematical model of the traditional APF method. In Section 3, the description of the modified approaches is presented to solve inherent problems in the traditional APF method. Meanwhile, we present the path planning strategy based on the modified APF approaches. In Section 4, the conducted experiments supported with a complete simulation of static obstacles, moving obstacles, and dynamic environment, as well as the experimental results, are provided. In Section 5, the practical application of the proposed method has been analyzed through a real environment experiment. Finally, conclusions are drawn in Section 6.

\section{Theoretical Foundations}

2.1. Attractive Potential Field Function. Set the current position coordinates of AUV as $X=(x, y)^{T}$, and the goal point coordinates as $X_{g}=\left(x_{g}, y_{q}\right)^{T}$. The AUV has been affected all along by the attractive force before reaching the goal point. The farther the goal point, the bigger the attraction. The attraction becomes zero when the AUV reaches the goal point. The attractive potential field $U_{\text {att }}$ produced by the goal point is

$$
U_{\text {att }}(X)=\frac{1}{2} k_{\mathrm{att}} \cdot \mathrm{d}^{2}\left(X, X_{g}\right),
$$

where $\mathrm{d}\left(X, X_{g}\right)=\left\|X_{g}-X\right\|$ is the Euclidean distance between the AUV and the goal point. $k_{\text {att }}$ is the attractive potential field constant.

The attractive force $F_{\text {att }}(X)$ of the AUV in the attractive potential field is the negative gradient of $U_{\text {att }}(X)$ :

$$
F_{\text {att }}(X)=-\nabla U_{\text {att }}(X)=k_{\text {att }} \cdot \mathrm{d}\left(X, X_{g}\right) .
$$

The direction of the attractive force is along the line between the AUV and the goal point and points to the goal point.
2.2. Repulsive Potential Field Function. Similar to the attractive potential field function, the repulsive potential field function is also distance-based. When the AUV is inside the influence range of the obstacle, it would be affected by the repulsion. The closer the obstacle, the bigger the repulsion. Set the coordinates of the obstacle as $X_{o}=\left(x_{o}, y_{o}\right)^{T}$; the repulsive potential field $U_{\text {rep }}(X)$ generated by the relative distance between the AUV and the obstacle is

$$
U_{\text {rep }}(X)= \begin{cases}\frac{1}{2} k_{\text {rep }}\left(\frac{1}{\mathrm{~d}\left(X, X_{o}\right)}-\frac{1}{d_{0}}\right)^{2}, & \mathrm{~d}\left(X, X_{o}\right) \leq d_{0}, \\ 0, & \mathrm{~d}\left(X, X_{o}\right)>d_{0},\end{cases}
$$

where $\mathrm{d}\left(X, X_{o}\right)=\left\|X_{o}-X\right\|$ is the Euclidean distance between the AUV and obstacle. $k_{\text {rep }}$ is the repulsive potential field constant. $d_{0}$ is the influence range of the repulsive potential field.

The repulsive force $F_{\text {rep }}(X)$ of the AUV in the repulsive potential field is the negative gradient of $U_{\text {rep }}(X)$ :

$$
\begin{aligned}
F_{\text {rep }}(X) & =-\nabla U_{\text {rep }}(X) \\
& = \begin{cases}k_{\text {rep }}\left(\frac{1}{\mathrm{~d}\left(X, X_{o}\right)}-\frac{1}{d_{0}}\right) \frac{\nabla \mathrm{d}\left(X, X_{o}\right)}{\mathrm{d}^{2}\left(X, X_{o}\right)}, & \mathrm{d}\left(X, X_{o}\right) \leq d_{0}, \\
0, & \mathrm{~d}\left(X, X_{o}\right)>d_{0} .\end{cases}
\end{aligned}
$$

The direction of the repulsive force is along the line between the AUV and obstacle and points to the AUV.

2.3. Resultant Potential Field Function. AUV will be affected by the combined action of the attractive potential field and the repulsive potential field while moving toward the goal point. The resultant potential field function $U(X)$ of AUV is

$$
U(X)=U_{\text {att }}(X)+U_{\text {rep }}(X)
$$

The resultant force $F(X)$ is

$$
\begin{aligned}
F(X) & =-\nabla U(X)=-\nabla U_{\text {att }}(X)-\nabla U_{\text {rep }}(X) \\
& =F_{\text {att }}(X)+F_{\text {rep }}(X)
\end{aligned}
$$

The establishment of the resultant force model in dynamic two-dimensional space is shown in Figure 1. $F_{\text {rep } 1}$ and $F_{\text {rep2 }}$ are the repulsive forces generated by the two obstacles, respectively. $F_{\text {rep }}$ is the total repulsive force from all obstacles. $F_{\text {att }}$ is the attractive force generated by the goal point. The resultant force $F$ can be obtained by the principle of superposition.

\section{Materials and Methods}

The APF method is here modified significantly to make it more suitable for AUV path planning in static and dynamic environments. Meanwhile, we propose the obstacle avoidance strategy based on the modified APF method. Table 1 shows the decision-making framework of the proposed path 


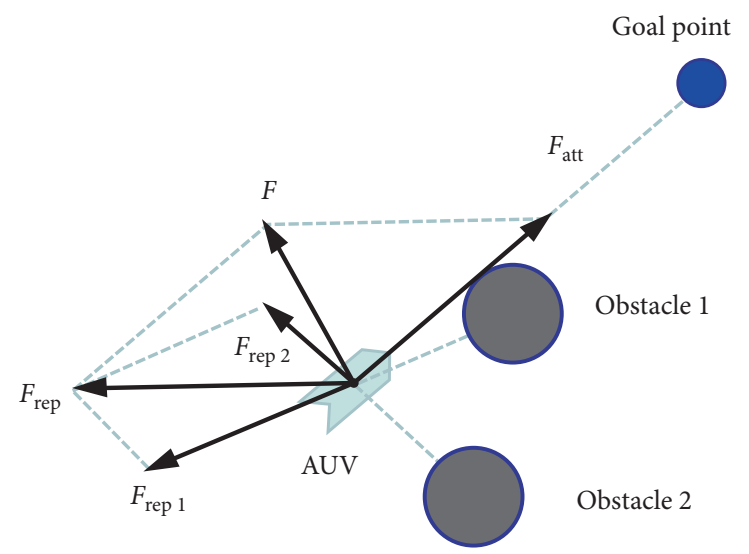

FIGURE 1: The resultant force model of AUV.

TABLE 1: The decision-making framework of the proposed path planning method.

\begin{tabular}{lccc}
\hline Environment & GNRON problem & Local minima problem & Both \\
\hline Static environment & 1 & 2 & No \\
Dynamic environment & 1,3 & 2,3 & 4,2 \\
\hline
\end{tabular}

* 1 : the distance correction factor method; 2: the RHG method; 3: the relative velocity method; 4: the APF method.

planning method. Figure 2 shows the flowchart of the proposed method. A distance correction factor is added to the original repulsive potential field function to solve the GNRON problem. The regular hexagon-guided (RHG) method is combined with the APF method to help the AUV escaping from the local minimum region. The relative velocity method adds the velocity factor to the APF method to solve the dynamic environment obstacle avoidance problem. AUV can choose an appropriate obstacle avoidance strategy through apperceiving outside environment and self-state and the motion of obstacles.

3.1. The Improvement of the GNRON Problem. The essential cause of the GNRON problem is that the goal point is not the minimum of the total potential function. From (3), if AUV infinite closes to the obstacle, $\mathrm{d}\left(X, X_{o}\right)$ approaches zero and $1 / d\left(X, X_{o}\right)$ approaches $\infty$. That is, as the AUV closes to the goal, on the one hand, the attractive potential field decreases; on the other hand, the repulsive potential field increases quickly. Figure 3(a) shows the GNRON problem in twodimensional space. The dotted circle represents the influence range of the repulsive potential field. When AUV is located at the position of Figure 3(a), it is affected by the combined action of $F_{\text {att }}$ and $F_{\text {rep }}$. The repulsion is much larger than the attraction, much like the principle of flow, moving from the higher potential filed to the lower potential field. The total force points to the direction away from the goal point. AUV cannot reach the goal point.

To solve the GNRON problem, a distance correction factor is added to the repulsive potential field function. It can balance the changes in two kinds of force, especially the rapid increase of the repulsive force. In this way, the repulsive force can be reduced gradually when the AUV closes to the goal point. It can also guarantee the resultant potential field at the goal point is the global minimum. With the attractive potential field unchanged, the repulsive potential field function can be defined as follows:

$$
U_{\text {rep }}(X)= \begin{cases}\frac{1}{2} k_{\text {rep }}\left(\frac{1}{\mathrm{~d}\left(X, X_{o}\right)}-\frac{1}{d_{0}}\right)^{2} \mathrm{~d}^{n}\left(X, X_{g}\right), & \mathrm{d}\left(X, X_{o}\right) \leq d_{0}, \\ 0, & \mathrm{~d}\left(X, X_{o}\right)>d_{0},\end{cases}
$$

where $d^{n}\left(X, X_{g}\right)$ is added to (7) based on (3). $d^{n}\left(X, X_{g}\right)$ is the distance between the AUV and the goal position. $n$ is an arbitrary real number which is greater than zero. The improved repulsive force function is given as follows:

$$
\begin{aligned}
& F_{\text {rep }}(X)=-\nabla U_{\text {rep }}(X) \\
& = \begin{cases}F_{\text {rep1 }}(X)+F_{\text {rep2 }}(X), & \mathrm{d}\left(X, X_{o}\right) \leq d_{0}, \\
0, & \mathrm{~d}\left(X, X_{o}\right)>d_{0} .\end{cases} \\
& F_{\text {rep1 }}(X) \text { and } F_{\text {rep2 }}(X) \text { are defined as } \\
& F_{\text {rep } 1}(X)=k_{\text {rep }}\left(\frac{1}{\mathrm{~d}\left(X, X_{o}\right)}-\frac{1}{d_{0}}\right) \frac{\mathrm{d}^{n}\left(X, X_{g}\right)}{\mathrm{d}^{2}\left(X, X_{o}\right)}, \\
& F_{\text {rep2 }}(X)=\frac{n}{2} k_{\text {rep }}\left(\frac{1}{\mathrm{~d}\left(X, X_{o}\right)}-\frac{1}{d_{0}}\right)^{2} \mathrm{~d}^{n-1}\left(X, X_{g}\right) .
\end{aligned}
$$

The resultant force model of the improved repulsive potential field is shown in Figure 3(b). The direction of the improved repulsive force $F_{\text {rep }}$ is not on the line between the AUV and the obstacle. This is different from the traditional APF method. $F_{\text {rep } 1}$ is one component of $F_{\text {rep }}$. Its direction is on the line between the AUV and the obstacle, and it makes AUV moving away from obstacles. $F_{\text {rep2 }}$ is another component of 


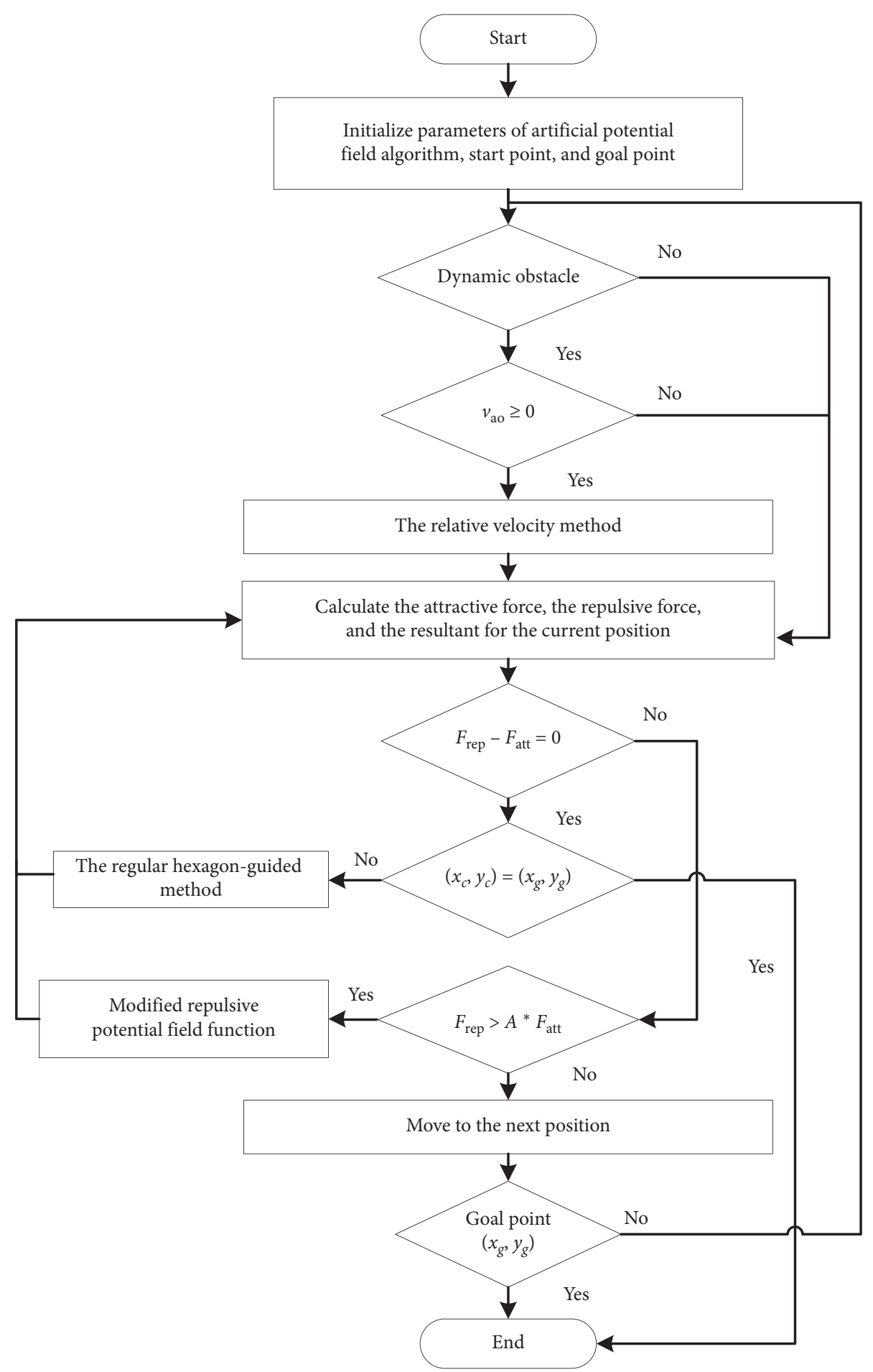

FIgURE 2: The flowchart of AUV path planning based on the improved APF method.

$F_{\text {rep }}$. Its direction is on the line between the AUV and the goal point, and it guides AUV to move toward the goal point.

The force condition of the AUV can make a further analysis based on the value of $n$ :

(1) When $n=1, F_{\text {rep1 }}(X)$ and $F_{\text {rep2 }}(X)$ can be expressed as follows:

$$
\begin{aligned}
& F_{\text {rep1 }}(X)=k_{\text {rep }}\left(\frac{1}{\mathrm{~d}\left(X, X_{o}\right)}-\frac{1}{d_{0}}\right) \frac{\mathrm{d}\left(X, X_{g}\right)}{\mathrm{d}^{2}\left(X, X_{o}\right)}, \\
& F_{\text {rep2 }}(X)=\frac{n}{2} k_{\text {rep }}\left(\frac{1}{\mathrm{~d}\left(X, X_{o}\right)}-\frac{1}{d_{0}}\right)^{2},
\end{aligned}
$$




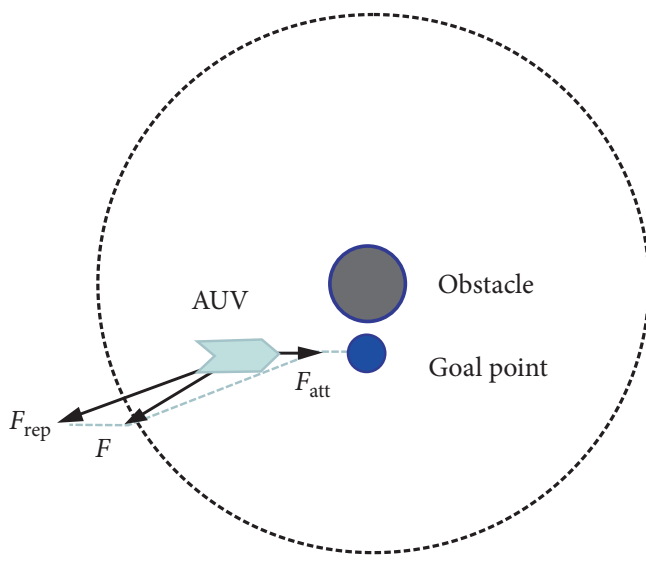

(a)

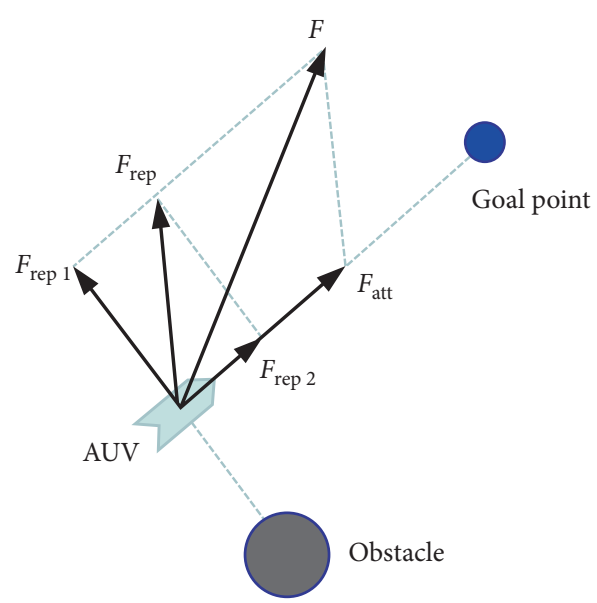

(b)

Figure 3: The improvement of the GNRON problem. (a) The GNRON problem of the traditional APF method. (b) The improved resultant force model.

when the AUV closes to the goal point, $\mathrm{d}\left(X, X_{g}\right)$ decreases and approaches zero. $F_{\text {repl }}$ approaches zero also and $F_{\text {rep } 2}$ is a constant. AUV moves toward the goal point under the action of attraction only.

(2) When $n>1$, with the AUV closes to the goal point, $\mathrm{d}^{n}\left(X, X_{g}\right)$ and $\mathrm{d}^{n-1}\left(X, X_{g}\right)$ approach zero. $F_{\text {repl } 1}$, $F_{\text {rep2 }}$, and the total repulsion $F_{\text {rep }}$ approach zero. AUV can reach the goal point gradually under the influence of the attractive force.

3.2. The Improvement of the Local Minima Problem. The AUV should stop moving after reaching the goal point because the resultant force is zero or minimized there. However, AUV may arrive at other locations where the attractive force and repulsive force are almost equal in collinear but opposite direction. The resultant force of the AUV is also likely to be zero or minimized. In this situation, the AUV is trapped in a local minimum region. Three common cases of local minima problems are shown in Figures 4(a)-4(c). The AUV, obstacle, and the goal point are in collinear in Figures 4(a) and 4(b). In the beginning, the attractive force is greater than the repulsive force. AUV moves toward the goal point along the line. The repulsive force increases with the distance between the AUV and the obstacle decreases. There necessarily exists a certain position where the repulsive force $F$ is zero and makes the AUV standstill. The local minima problem with multiple obstacles is shown in Figure 4(c). The attractive force is offset by the repulsive force so that AUV entirely in stop motion.

For solving the local minima problem, we propose a regular hexagon-guided (RHG) method. As shown in Figures 4(d) and 4(e), we assume that AUV trapped in the local minimum region. Let us say that the local minima point is the coordinate origin. Use the line where the AUV and the goal point are connected as the $y$-axis. Take the line passing through the origin point and perpendicular to the $y$-axis as the $x$-axis. Construct a virtual regular hexagon in which the distance of a one-step position is taken as the side length.
When the AUV traps in a local minimum region, an angle $\theta$ is generated along the $x$-axis. $F_{\theta}$ is the motion direction of the next step size, which can help the AUV escaping from the local minimum region. When $\theta$ is positive, AUV rotates $90-$ $|\theta|$ degrees clockwise. When $\theta$ is negative, AUV rotates $90-$ $|\theta|$ degrees counterclockwise. Each interior angle of a regular hexagon is 120 degrees, so angle $\theta$ is a constant, $\theta=30$. AUV can move along the virtual regular hexagon that is close to the target point but not in the obstacle area to get rid of the local minimum region. If the target point is within the obstacle range, the RHG method will be stopped when the distance of the AUV from the target point is less than the distance from the obstacle.

To ensure that the AUV can successfully pick out the local minima at once and improve the efficiency of navigation, we do further analysis of the RHG method.

(1) As shown in Figure 4(f), the AUV is located at the local minimum region where the obstacles are placed symmetrically. AUV scans the surrounding obstacles using sensors and locates the obstacles. Construct a two-dimensional coordinate system and a virtual regular hexagon. If the area of the obstacle is large, some new regular hexagons can be added to the first regular hexagon, as the cellular mobile network. AUV moves along the position of the regular hexagon until it gets far away from the obstacle.

(2) When the AUV encounters a concave-shaped obstacle, local minimum occurs, and as a result, the AUV runs in a closed loop, as shown in Figure 4(g). The AUV moves horizontally backwards or turns around if that is possible. Once it reaches sufficiently away from the local minimum region, the AUV builds a two-dimensional coordinate system and a virtual regular hexagon. AUV constructs the guidance framework by detecting and locating obstacles, thus getting rid of the local minimum region. The best obstacle avoidance path can be found. 


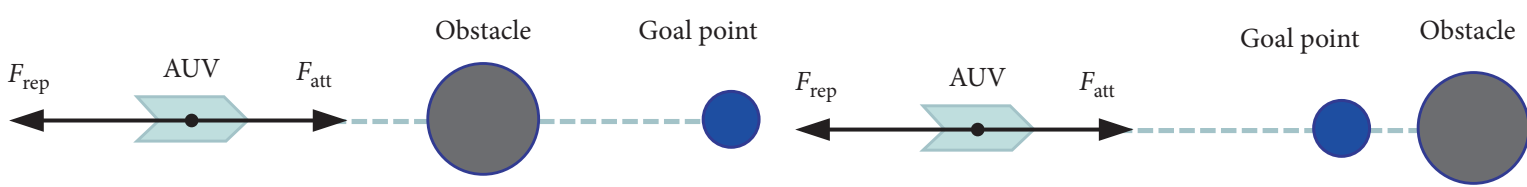

(a)

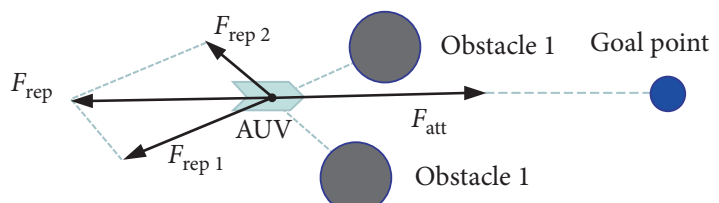

(c)

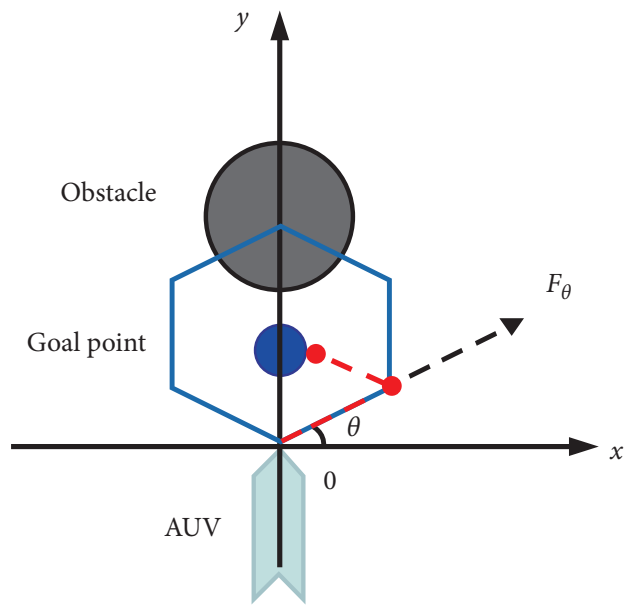

(e) (b)

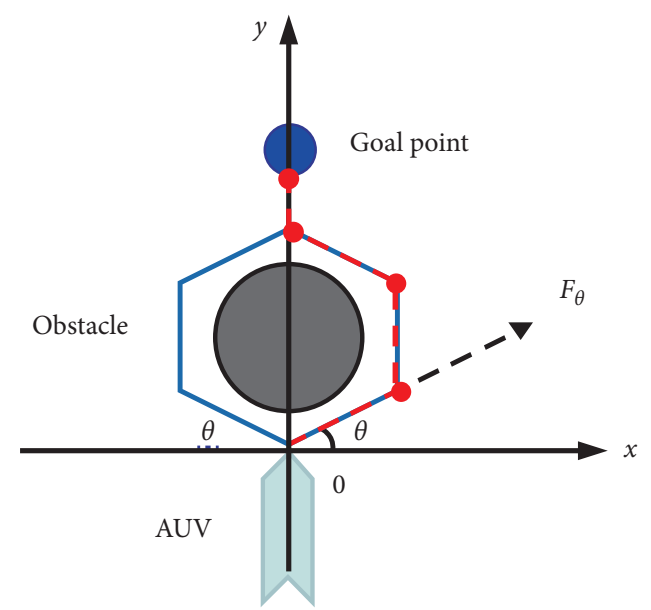

(d)

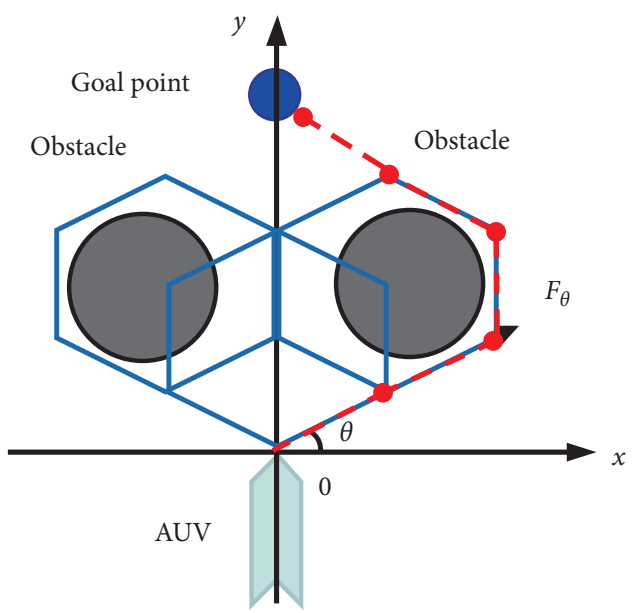

(f)

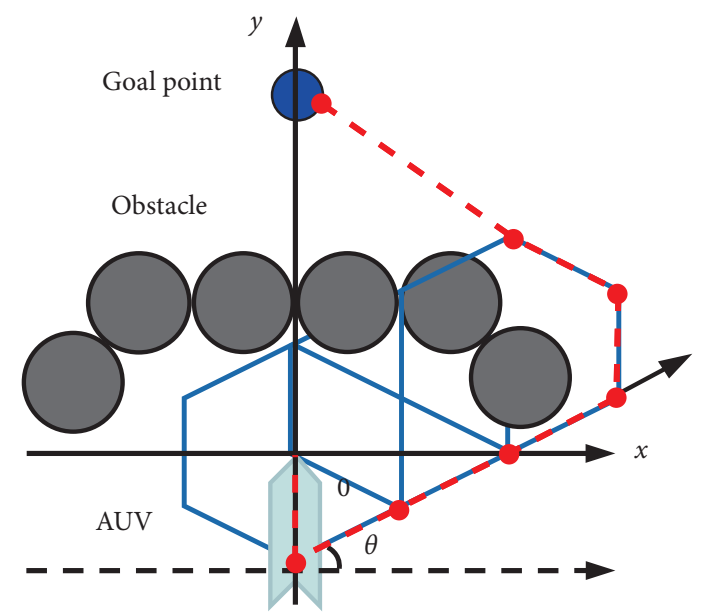

(g)

FIgURE 4: The improvement of the local minima problem. (a) Local minimum problem with three points in collinear of the traditional APF method (obstacle is between the AUV and target point). (b) Local minimum problem with three points in collinear (target point is between the AUV and obstacle). (c) The local minimum region with multiple obstacles. (d) The improvement of the case (a). (e) The improvement of the case (b). (f) The improvement of the case (c). (g) The improvement of concave shape obstacle. 
It can be seen from the above analysis that the RHG method has two definite advantages. On the one hand, this method can avoid the appearance of foldback and oscillations because of the improper parameter selection. On the other hand, this method significantly reduces the calculation burden through the fixed parameter.

\subsection{The Improvement of the Dynamic Environment Obstacle} Avoidance Problem. When the velocity of the AUV and moving obstacles are slow, path planning can determine by the traditional APF method. When the velocity of the AUV or moving obstacles or both are fast, AUV could collide with moving obstacles by the traditional APF method. The reason is that the traditional APF method applies to static obstacles and has no regard for the effect of moving velocity. When AUV deviates from moving obstacles, the traditional APF method takes the measure to avoid the obstacles still. As a result, the path becomes longer, and the efficiency of navigation is reduced.

As shown in Figure 5(a), the AUV is affected by the repulsive field at $t_{0}$. It begins to change course by calculating the resultant force. At $t_{4}$, AUV has rightly figured out the position of the next step size. But AUV has not changed course in time because of the high speed, which could pose a collision risk. As shown in Figure 5(b), the AUV enters the influence range of the moving obstacle at $t_{0}$ and changes course. Actually, the moving obstacle is moving away from the AUV. AUV shall soon outside the influence range of the obstacle and moves toward the goal point under the action of the attractive force only. Even if the AUV does not change course, there is no risk of collision.

To solve the dynamic environment obstacle avoidance problem, the APF method must consider not only the spatial location but also the magnitude and direction of the velocity of the moving obstacles. In this paper, we propose the relative velocity method. We further improve the repulsive potential field function $U_{\text {rep }}(X)$ based on (7). Take advantage of the relative velocity component between the AUV and moving obstacle in the direction from the AUV to the obstacle as the judgment of obstacle avoidance. The new repulsive potential field function $U_{\text {rep }}(X, V)$ is defined as follows:

$$
U_{\text {rep }}(X, V)= \begin{cases}U(X)+U(V), & \mathrm{d}\left(X, X_{o}\right) \leq d_{0} \& v_{\mathrm{ao}} \geq 0, \\ 0, & \text { else, }\end{cases}
$$

where

$$
\begin{aligned}
& U(X)=\frac{1}{2} k_{\mathrm{rep}}\left(\frac{1}{\mathrm{~d}\left(X, X_{o}\right)}-\frac{1}{d_{0}}\right)^{2} \mathrm{~d}^{n}\left(X, X_{g}\right), \\
& U(V)=k_{v} \frac{\left(v-v_{o}\right)^{T} e_{\mathrm{ao}}}{\mathrm{d}\left(X, X_{o}\right)}=k_{v} \frac{v_{\mathrm{ao}}}{\mathrm{d}\left(X, X_{o}\right)},
\end{aligned}
$$

where $v_{o}$ is the velocity of moving obstacles. $v$ is the velocity of the AUV. If $v_{o} \geq v$, AUV plans a path behind the obstacle. If $v_{o}<v$, AUV plans a path in front of the obstacle. $U(X)$ is the same as $U_{\text {rep }}(X)$ in $(7) . U(V)$ is the velocity repulsive potential field produced by moving obstacle. $k_{v}$ is the velocity repulsive potential field constant. $e_{\mathrm{ao}}$ is the acceleration repulsive potential field vector pointing from the AUV to the moving obstacle. $\mathrm{d}\left(X, X_{o}\right)$ is the distance between the AUV and moving obstacle. $\left(v-v_{o}\right)^{T} e_{\mathrm{ao}}=v_{\mathrm{ao}}$ represents the relative velocity component between the AUV and the dynamic obstacle in the direction from the AUV to the obstacle. $v_{\mathrm{ao}} \geq 0$ shows the AUV moving toward the moving obstacles. $v_{\mathrm{ao}}<0$ shows the AUV deviation from the moving obstacle.

The improved repulsive force $F_{\text {rep }}(X, V)$ of the AUV in a dynamic environment is given as follows:

$$
\begin{aligned}
F_{\text {rep }}(X, V) & =-\nabla U_{\text {rep }}(X, V) \\
& = \begin{cases}F(X)+F(V), & \mathrm{d}\left(X, X_{o}\right) \leq d_{0} \& v_{\mathrm{ao}} \geq 0, \\
0, & \text { else, }\end{cases}
\end{aligned}
$$

where

$$
\begin{aligned}
F(X)= & -\nabla_{X} U(X)=F_{1}(X)+F_{2}(X) \\
= & k_{\text {rep }}\left(\frac{1}{\mathrm{~d}\left(X, X_{o}\right)}-\frac{1}{d_{0}}\right) \frac{\mathrm{d}^{n}\left(X, X_{g}\right)}{\mathrm{d}^{2}\left(X, X_{o}\right)} \\
& +\frac{n_{2}}{2} k_{\text {rep }}\left(\frac{1}{\mathrm{~d}\left(X, X_{o}\right)}-\frac{1}{d_{0}}\right)^{2} \mathrm{~d}^{n-1}\left(X, X_{g}\right), \\
F(V)= & -\nabla_{V} U(V)=-k_{v} \frac{v_{\text {ao }}}{\mathrm{d}\left(X, X_{o}\right)}=k_{v} \frac{v_{\text {oa }}}{\mathrm{d}\left(X, X_{o}\right)},
\end{aligned}
$$

where $F(X)$ is the same as $F_{\text {rep }}(X)$ in $(8) . F(V)$ is the velocity repulsive force. The improved resultant force model of AUV is shown in Figure 5(c).

The improved repulsive potential field function allows the AUV to gain greater repulsion when it has detected the moving obstacles. This can significantly improve its safety. Take advantage of $\left(v-v_{o}\right)^{T} e_{\mathrm{ao}}=v_{\mathrm{ao}}$ to judge the state of relative motion between the AUV and moving obstacle. So that blind obstacle avoidance can be avoided. The relative movement of the AUV and moving obstacle is shown in Figure 6. $l_{1}$ is the connection line between the AUV and the center of the obstacle, $l_{2}|| l_{1}$, and $l_{3} \perp l_{1} . v_{\text {ao }}$ is the component of the relative velocity in the direction of $l_{1}$. As shown in Figure 6(a), $v_{\text {ao }}$ points to the moving obstacle; that is, $v_{\mathrm{ao}} \geq 0$. AUV is moving toward the dynamic obstacle, and obstacle avoidance becomes an inevitable event. As shown in Figure 6(b), $v_{\text {ao }}$ points to the opposite direction of the moving obstacle; that is, $v_{\text {ao }}<0$. AUV is deviating from the dynamic obstacle, and there is no need for obstacle avoidance.

\section{Experiments and Analysis}

To evaluate the performance of the proposed method, the algorithm is verified on MATLAB R2017b, assuming that the AUV is a particle. The selection and setting of simulation parameters are according to the most disadvantage condition mentioned in Section 2. To make the simulations 


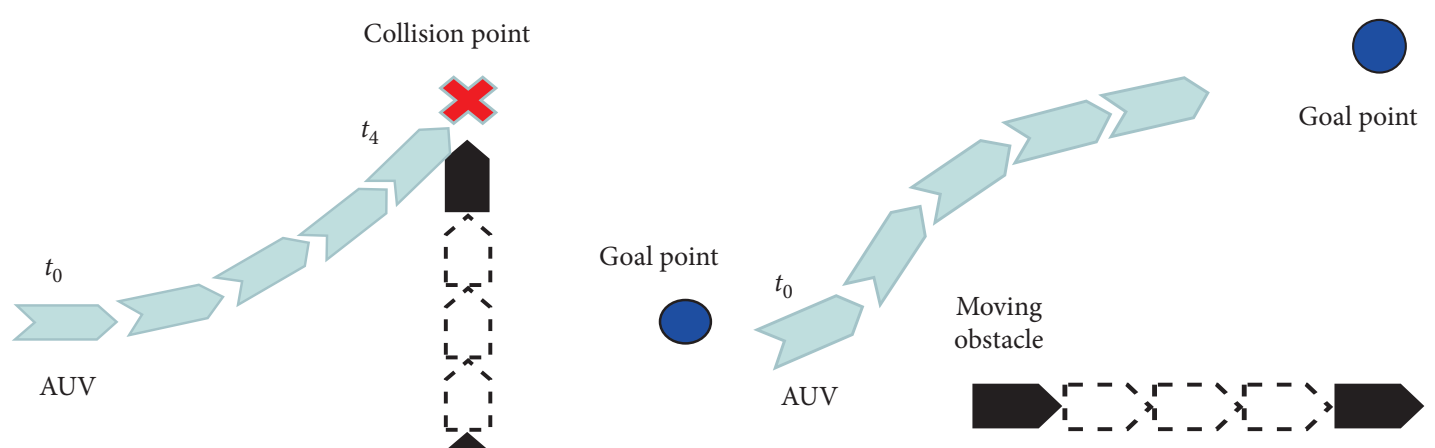

(a)

obstacle

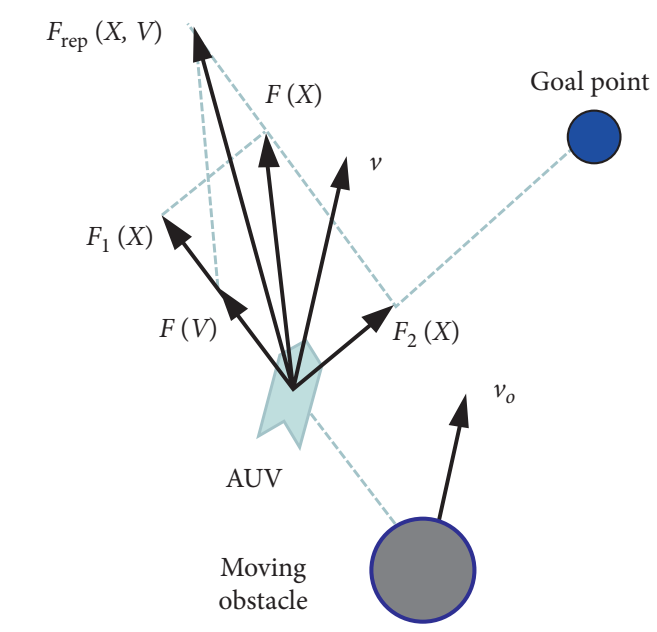

(b)

(c)

FIgURE 5: The improvement of the dynamic environment obstacle avoidance problem. (a) AUV collision with moving obstacle. (b) AUV deviation from moving obstacle. (c) The improved resultant force of moving obstacle avoidance problem.

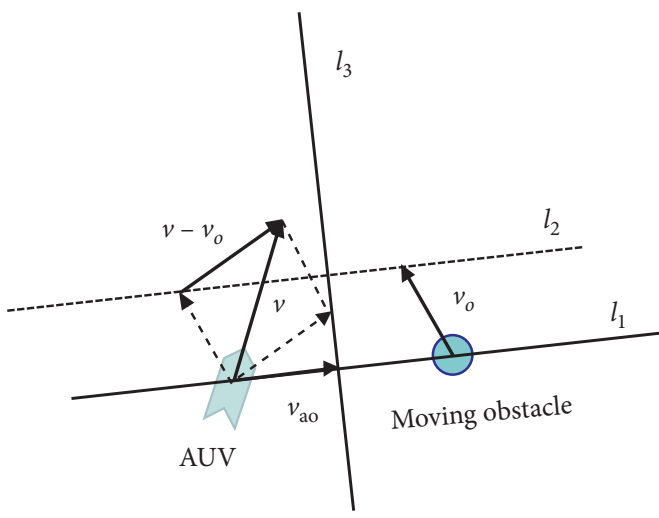

(a)

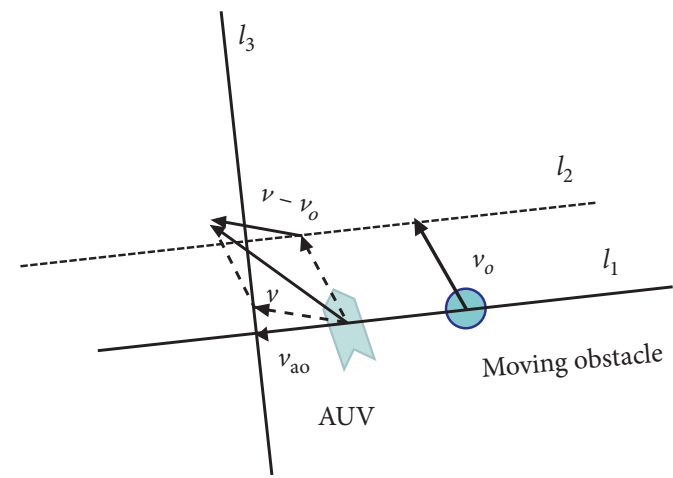

(b)

FIgURE 6: The relative motion of the AUV and moving obstacle. (a) AUV toward moving obstacle. (b) AUV deviation from moving obstacle.

simple, there are no uncertainties such as noises added to the simulated measured ranges between the AUV and obstacles. The attractive potential field constant is chosen as $k_{\text {att }}=40$. The repulsive potential field constant is chosen as $k_{\text {rep }}=1.25 \times 10^{5}$. We compare the proposed method to the traditional APF approach in the simulation environment to show how the RHG method can improve the performance of the APF and how the relative velocity method can help the AUV avoiding the moving obstacle.

4.1. Static Obstacles. In this section, there are four different cases considered for the simulation of the improved method. The cases are simulated with obstacles of equal sizes and they 
are located such that local minima occur in a free environment.

The initial position of AUV is $(0,0)^{T}$, and the goal point is $(200,200)^{T}$. The influence range of a single obstacle is set as $d_{0}=20$. The velocity of the AUV is set as $v=2 \mathrm{~m} / \mathrm{s}$ (the water velocity can be regarded as constant in a certain region and time). The position coordinates of the obstacles are fixed.

Figures 7(a) and 7(b) show the path generated by the proposed method for case 1 (as shown in Figures 4(a) and $4(\mathrm{~b}))$. The center position of obstacle is $(180,180)^{T}$. The start point, the obstacle center position, and the goal point are in collinear. The goal point is inside the influence range of the obstacle. AUV moves toward the goal point and traps in the local minimum region. The AUV cannot avoid the local minimum region using the traditional APF algorithm. A regular hexagon is generated through the RHG method. AUV gets the information of the next-step navigation point in the horizontal plane and then escapes from the local minimum region by moving to the next location. The AUV reaches the goal point successfully, and there are no foldback and oscillations.

Figures 7(c) and 7(d) show the path planning situation of the AUV due to the symmetry of the environment that is caused by nonconcave obstacles for case 2 (as shown in Figure $4(\mathrm{c}))$. The start point, the center coordinates of the obstacles, and the goal point are in collinear. The center positions of obstacles are $(140,140)^{T}$ and $(160,160)^{T}$ in Figure 7 (c). The center positions of obstacles are $(120,120)^{T}$, $(140,140)^{T}$, and $(160,160)^{T}$ in Figure $7(d)$. The AUV runs in a closed loop and could not reach the goal point using the traditional APF algorithm. The improved APF approach can get a smooth planned path along the edge of the influence range of obstacles.

Figure 7(e) shows a complicated environment with multiple obstacles of the same sizes for case 3 (as shown in Figure $4(\mathrm{f}))$. The center coordinates of obstacles are $(30,30)^{T}$, $(50,120)^{T},(120,50)^{T},(145,160)^{T}$, and $(160,145)^{T}$, which are symmetrical about centerline between the start point and the goal point. AUV moves toward the goal point. The second group of obstacles is symmetrical, and the AUV is outside the influence ranges of the obstacles. It is not affected by the repulsive potential field, and there is no need to avoid the obstacles. The third set of obstacles is symmetrical and attach tightly, and the two repulsive potential fields are superimposed on the AUV. The AUV traps in the local minimum region and cannot move further in the forward direction using the traditional APF algorithm. AUV detours the obstacle to the goal position by the regular hexagon-guided method, and there are a full path and no foldback.

Figure $7(\mathrm{f})$ shows the local minimum situation due to obstacles located in a concave for case 4 (as shown in Figure $4(\mathrm{~g})$ ). When using the traditional APF algorithm, AUV tries to avoid each obstacle. It finally gets into a closed loop and keeps running in the closed loop. AUV can escape from this local minimum condition with the improved APF method.

4.2. Moving Obstacles. The initial position of AUV is $(0,0)^{T}$, and the goal point is $(400,400)^{T}$. The influence range of a single obstacle is set as $d_{0}=40$. The velocity repulsion potential field constant is set as $k_{v}=1$. The acceleration repulsive potential field vector is set as $e_{\mathrm{ao}}=(1,0)^{T}$. The AUV starts moving from the initial position $(0,0)^{T}$ at constant velocity $v=2 \mathrm{~m} / \mathrm{s}$. In Figure 8 , there is the motion trajectory of the obstacles below the arrow, and the direction of the arrow indicates its direction of motion (under the assumption of line movement with constant velocity).

As evident from Figure 8(a), obstacle moves at velocity $v_{0}=(0.0,1.8)^{T}$ starting from point $(250,145)^{T}$. AUV enters the influence range of the moving obstacle. The relative velocity can be obtained through computation $v_{\mathrm{ao}} \geq 0$. That is, AUV is moving toward the dynamic obstacle. Meanwhile, the obstacle velocity is slower, and the AUV can plan a path in front of the obstacle. In contrast to the traditional APF method, the improved APF algorithm can generate a collision-free path for the AUV.

As shown in Figure 8(b), obstacle moves at velocity $v_{0}=$ $(0.0,1.8)^{T}$ starting from point $(300,210)^{T}$. Through the judgment of the relative velocity $v_{\text {ao }}<0$, AUV deviates from the moving obstacle, and there is no need for obstacle avoidance. The planned path using the improved APF method is more efficient than the one using the traditional APF method.

As shown in Figure 8(c), there are two moving obstacles. One moves at velocity $(1.8,0.0)^{T}$ starting from point $(210$, $100)^{T}$ and the other moves at velocity $(0.0,1.8)^{T}$ from point $(280,240)^{T}$. When entering the influence range of the moving obstacles, the AUV can change course and generate a collision-free path using the improved method.

As shown in Figure 8(d), there are two moving obstacles. One moves at velocity $v_{01}=(1.0,0.0)^{T}$ starting from point $(210,100)^{T}$ and the other moves at velocity $v_{02}=(1.414$, $1.414)^{T}$ from point $(240,200)^{T}$. The motion direction of the second moving obstacle is parallel with the AUV within the error range allowed, and the velocity of the obstacle is similar to AUV. AUV needs fine-tuning only and reaches the goal point using the proposed method. The relative velocity method generates a shorter path than the traditional method and provides safe obstacle avoidance.

4.3. Dynamic Environment. The simulation environment includes five static obstacles and a moving obstacle. The center coordinates of static obstacles are $(50,50)^{T},(50,160)$ $T,(160,50)^{T},(185,200)^{T}$, and $(200,185)^{T}$, which are symmetrical about centerline between the start point and the goal point. Moving obstacle moves at velocity $v_{0}=(0.0,1.8)^{T}$ starting from position $(280,140)^{T}$. Figure 9 shows the simulation result of the AUV obstacle avoidance. The proposed method can make decisions according to the AUV self-state and the motion of obstacles. It is clear that the AUV is capable of avoiding all the obstacles efficiently in contrast to the traditional APF method. The entire path trajectory of obstacle avoidance is complete without oscillations and foldback.

4.4. Analysis. The above simulation results have shown the effectiveness of the proposed algorithm in obstacle 


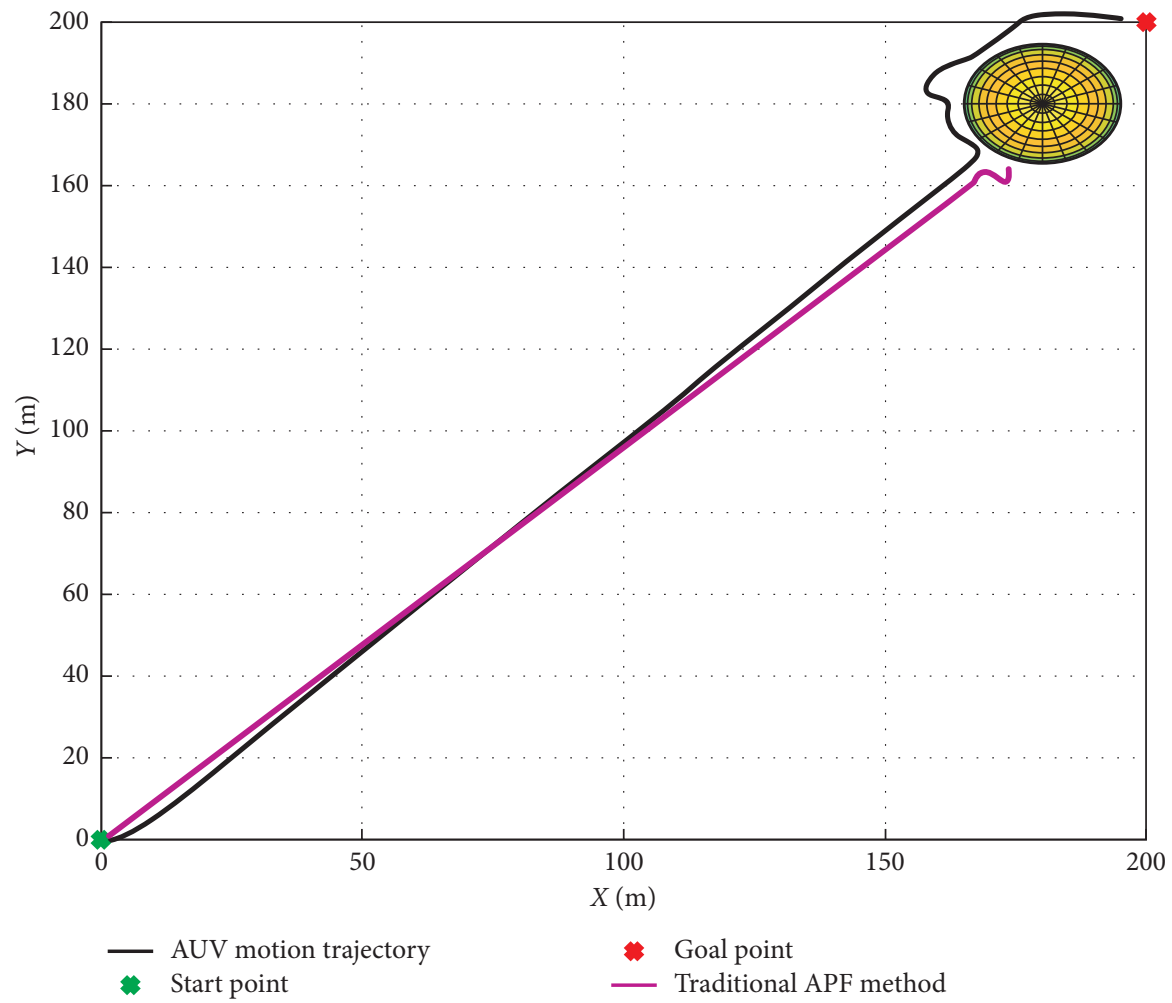

(a)

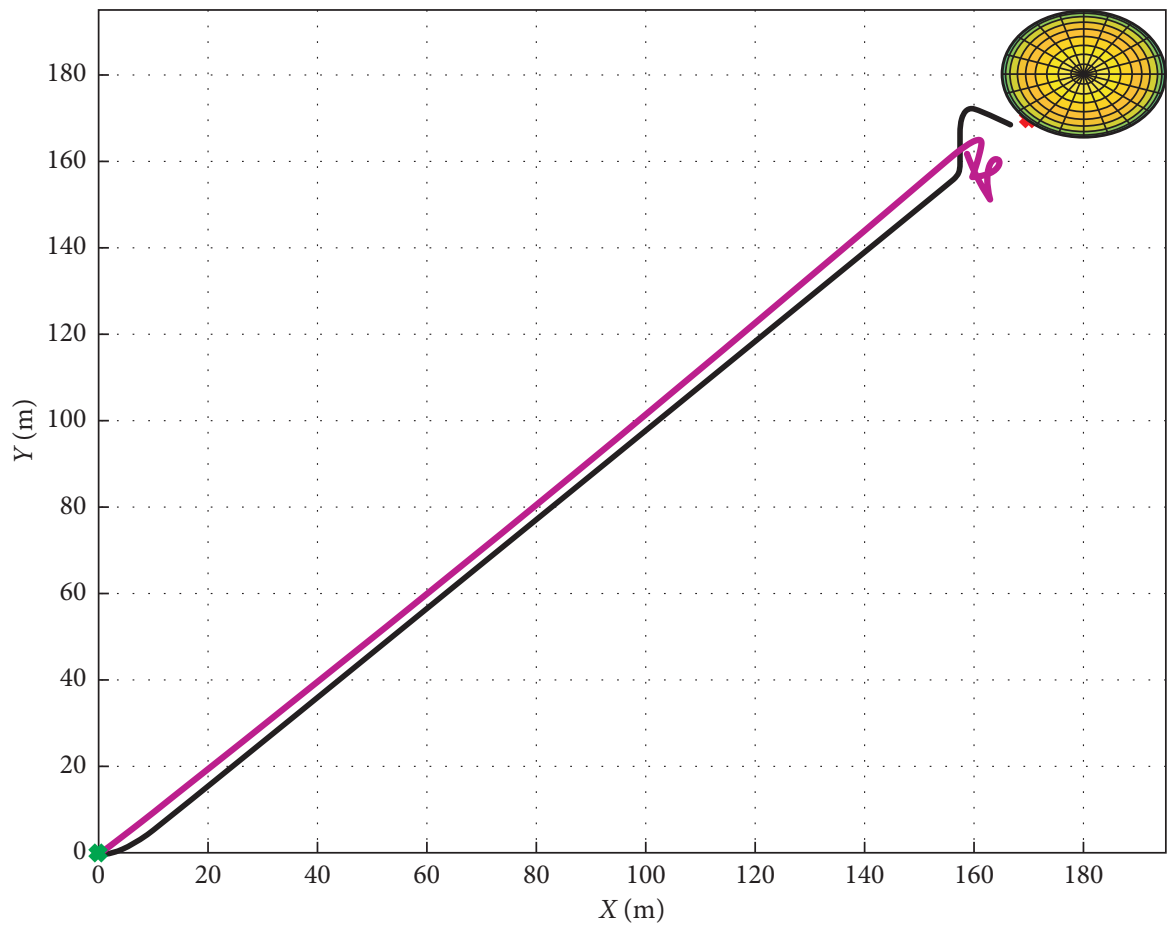

AUV motion trajectory

* Start point
* Goal point

— Traditional APF method

(b)

Figure 7: Continued. 


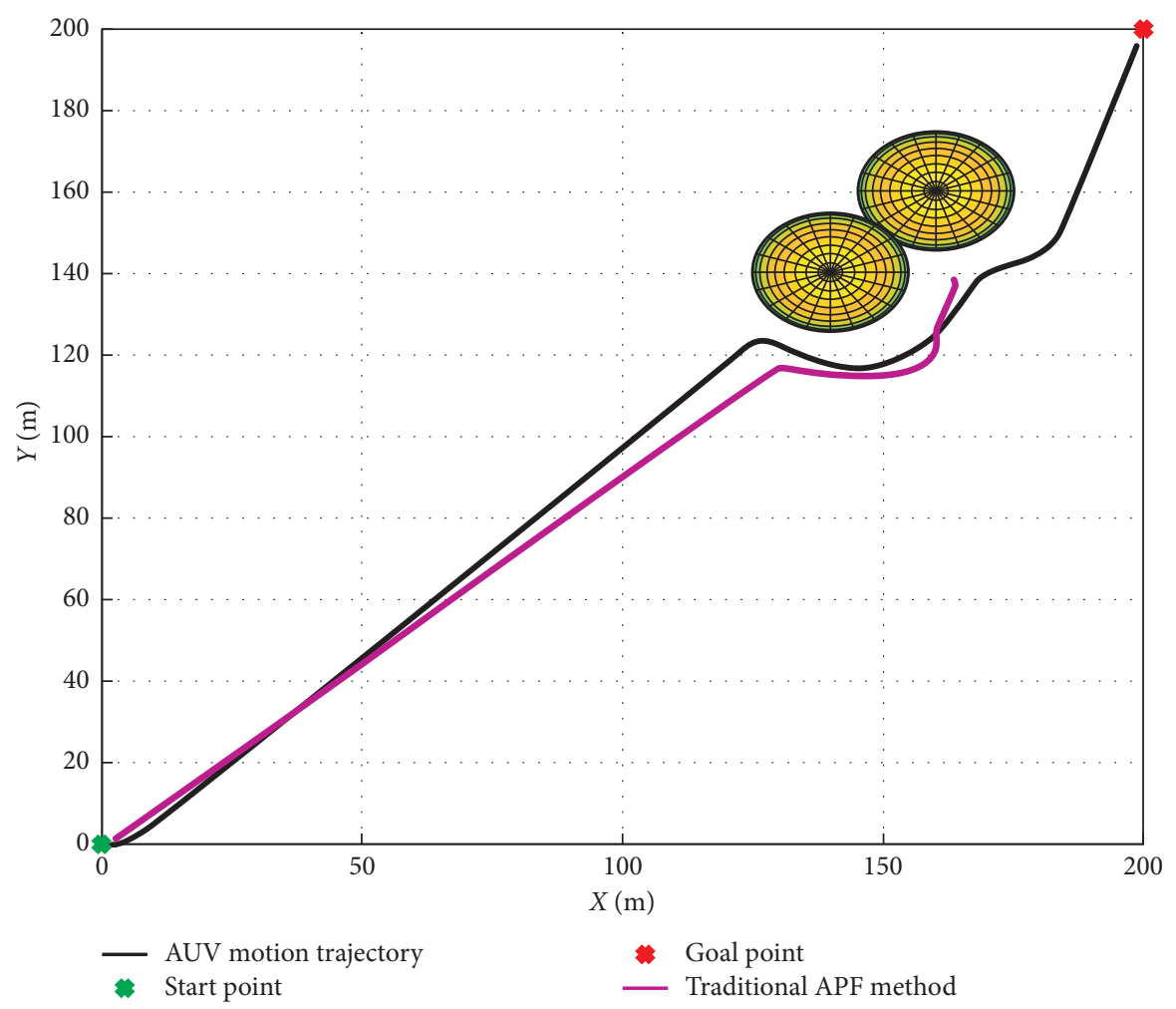

(c)

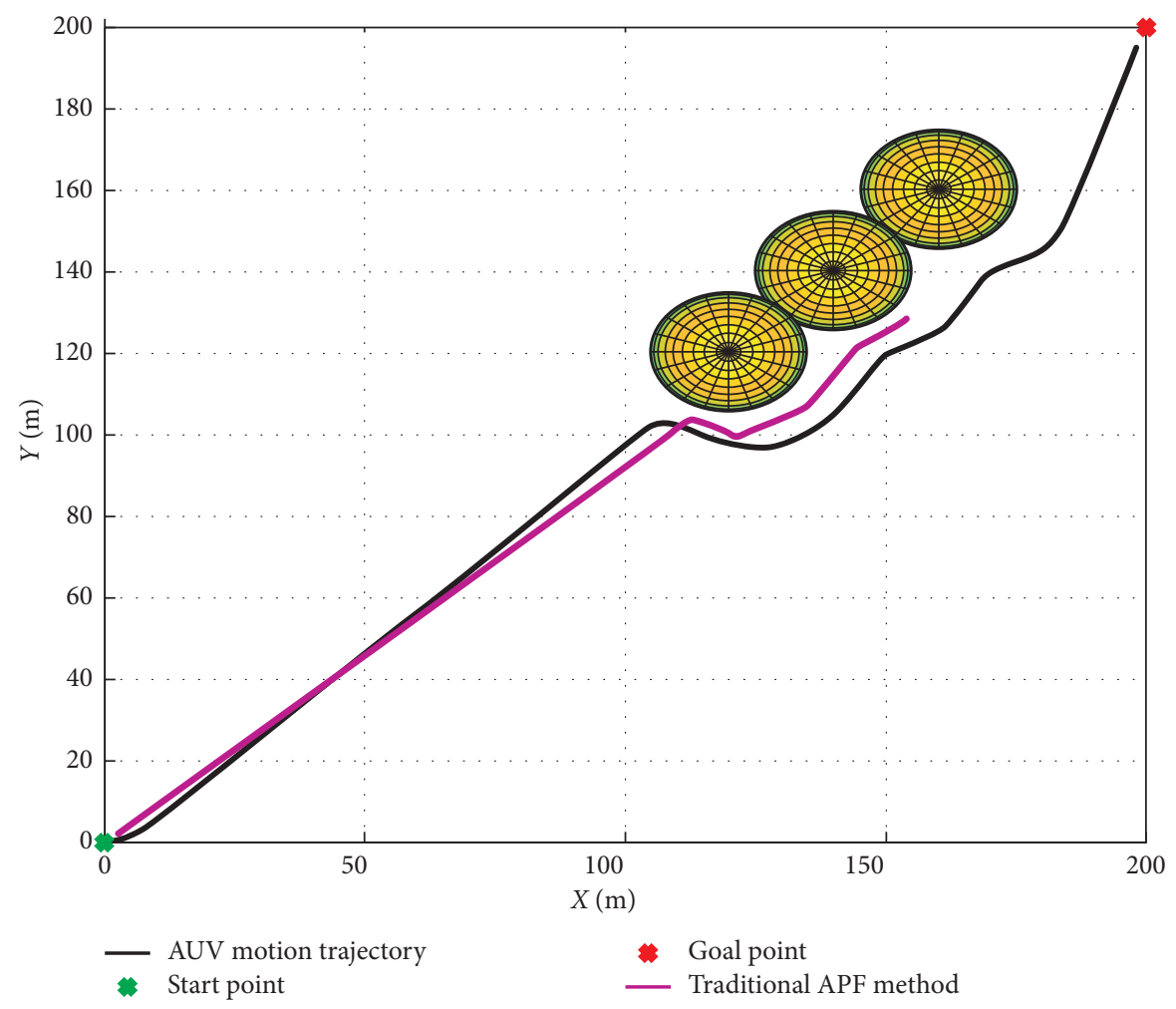

(d)

Figure 7: Continued. 


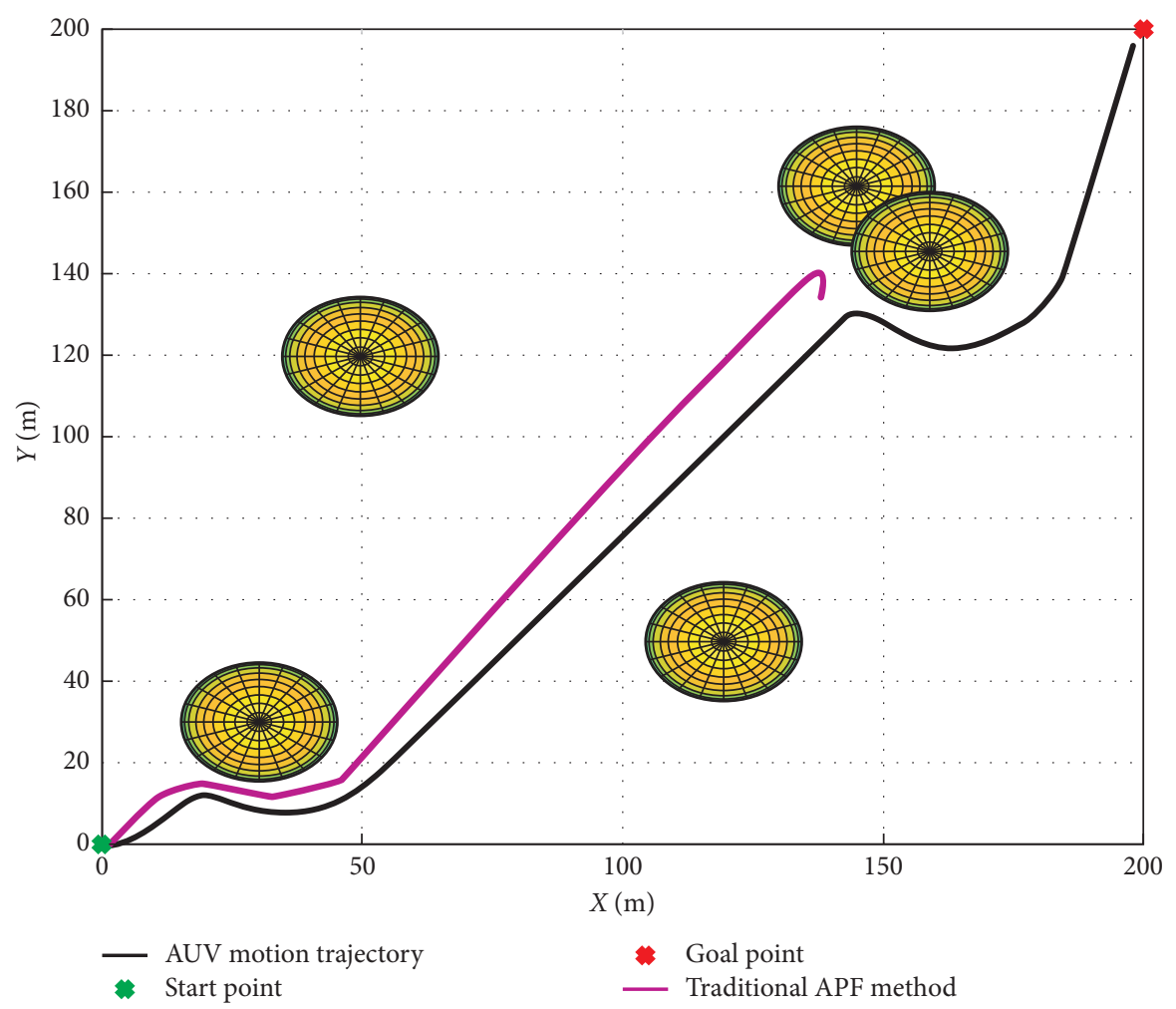

(e)

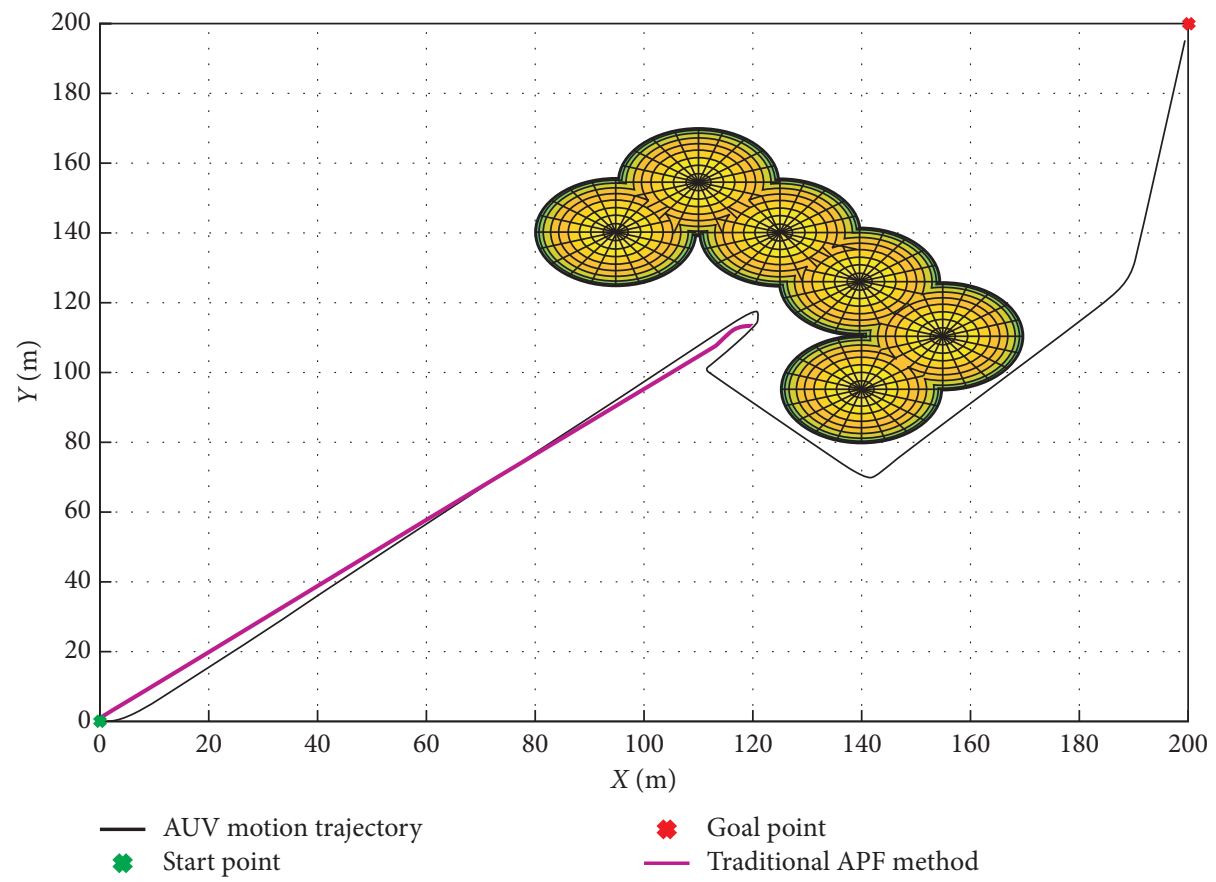

(f)

FiguRe 7: Experimental results of static obstacles. (a) The single obstacle (obstacle in the middle). (b) The single obstacle (the target point in middle). (c) The symmetry obstacles (two obstacles). (d) The symmetry obstacles (three obstacles). (e) Multiple obstacles. (f) Concave shape obstacle.

avoidance of AUV. Since the proposed path planning method involves the improvement of the APF method as well as the choice of obstacle avoidance strategy, it has definite advantages in finding a smooth, obstacle-free path for AUV. The RHG method can solve the local minima problems successfully, as shown in Figure 7. When AUV is inside the influence range of the obstacle, it can avoid obstacles and reach the goal point. When AUV is outside the 


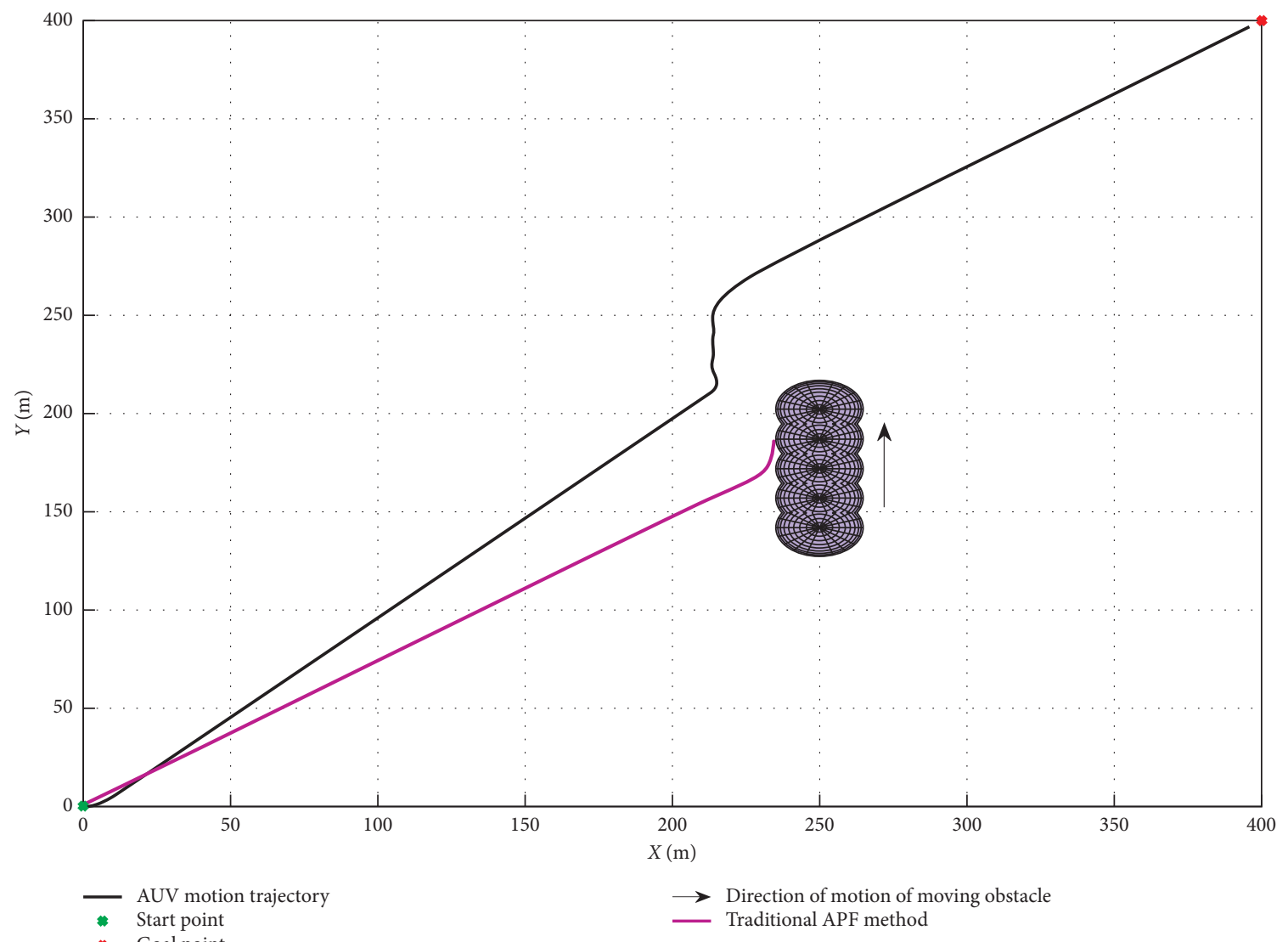

(a)

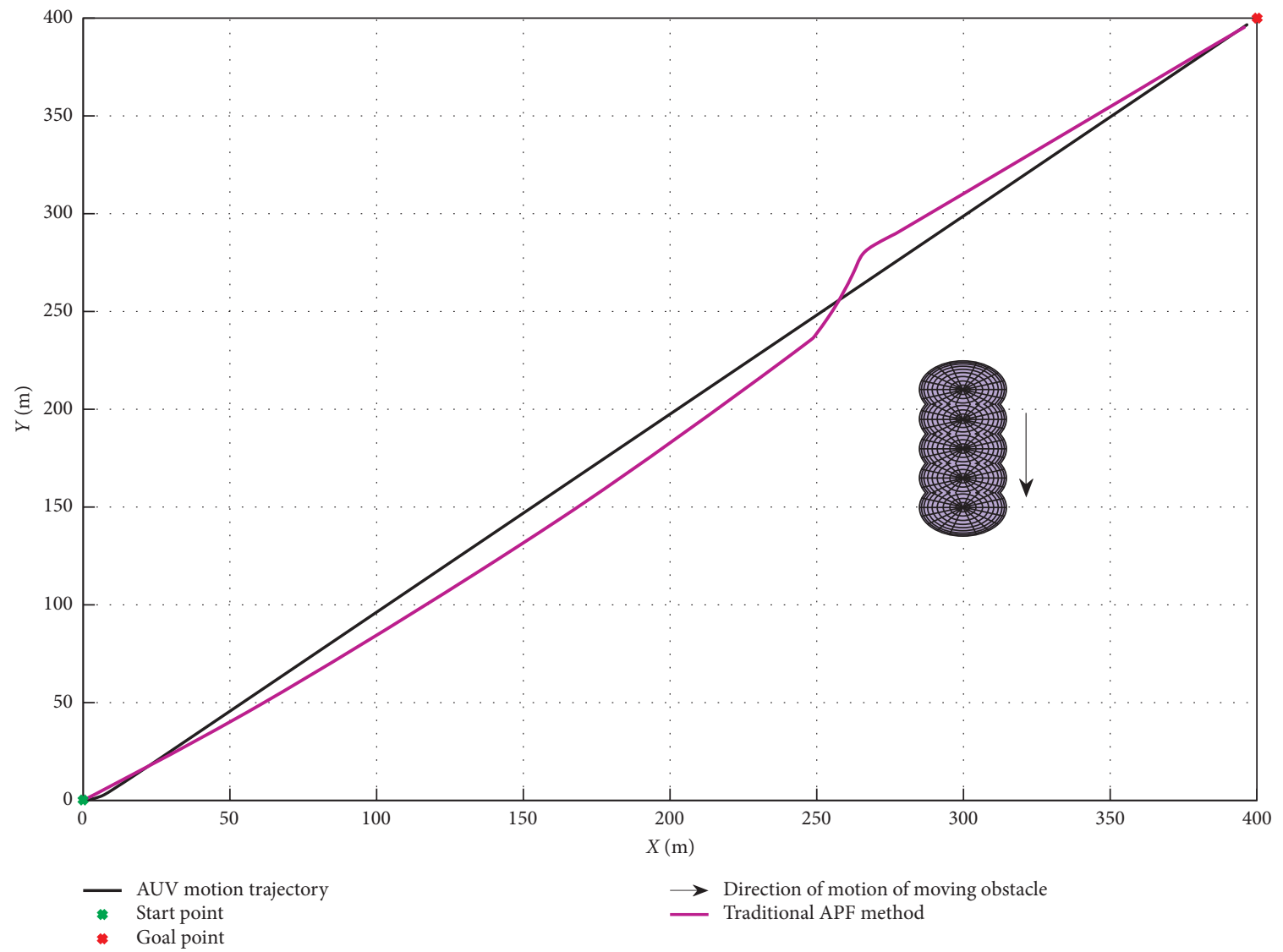

(b)

FIgURE 8: Continued. 


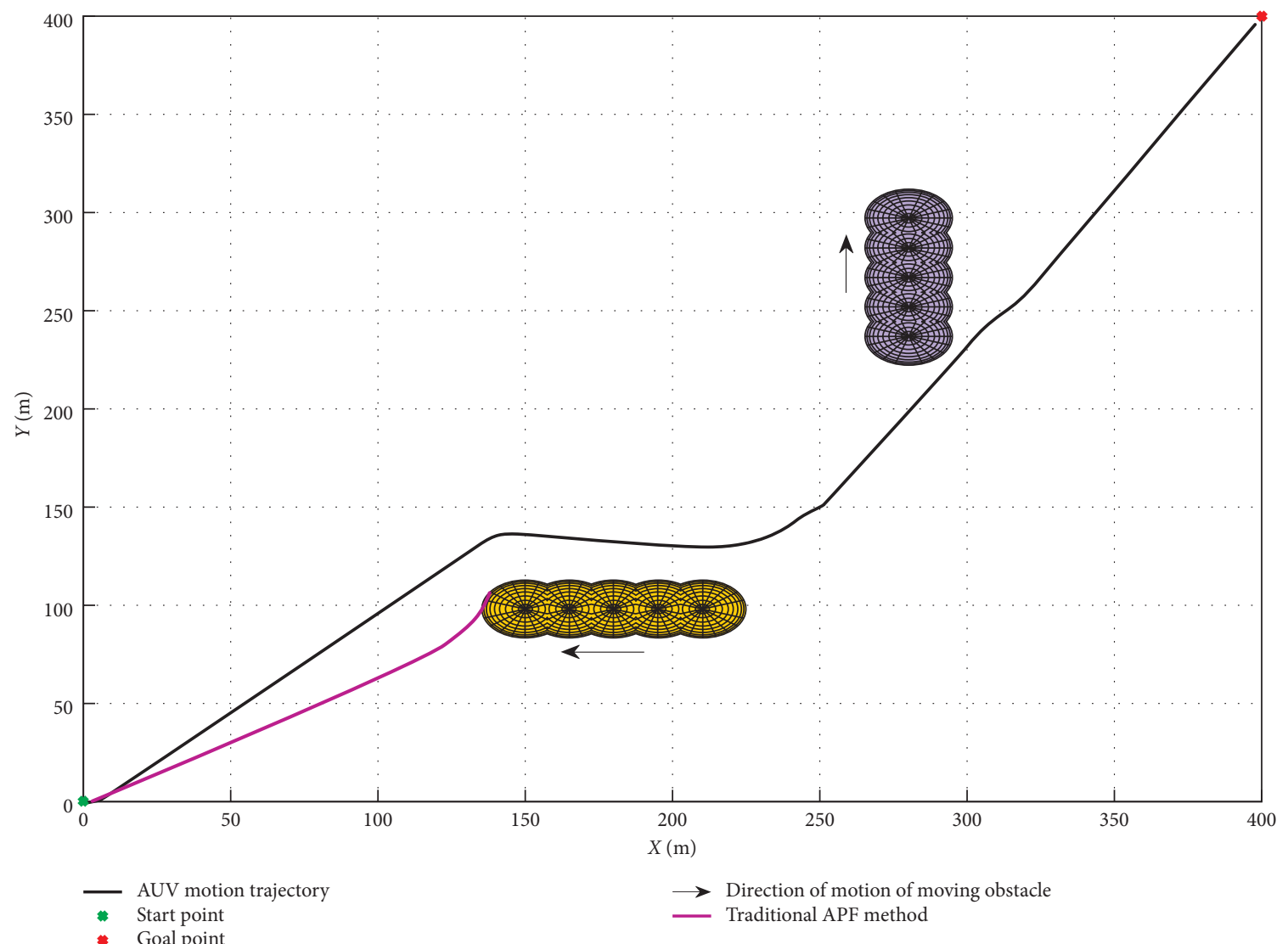

(c)

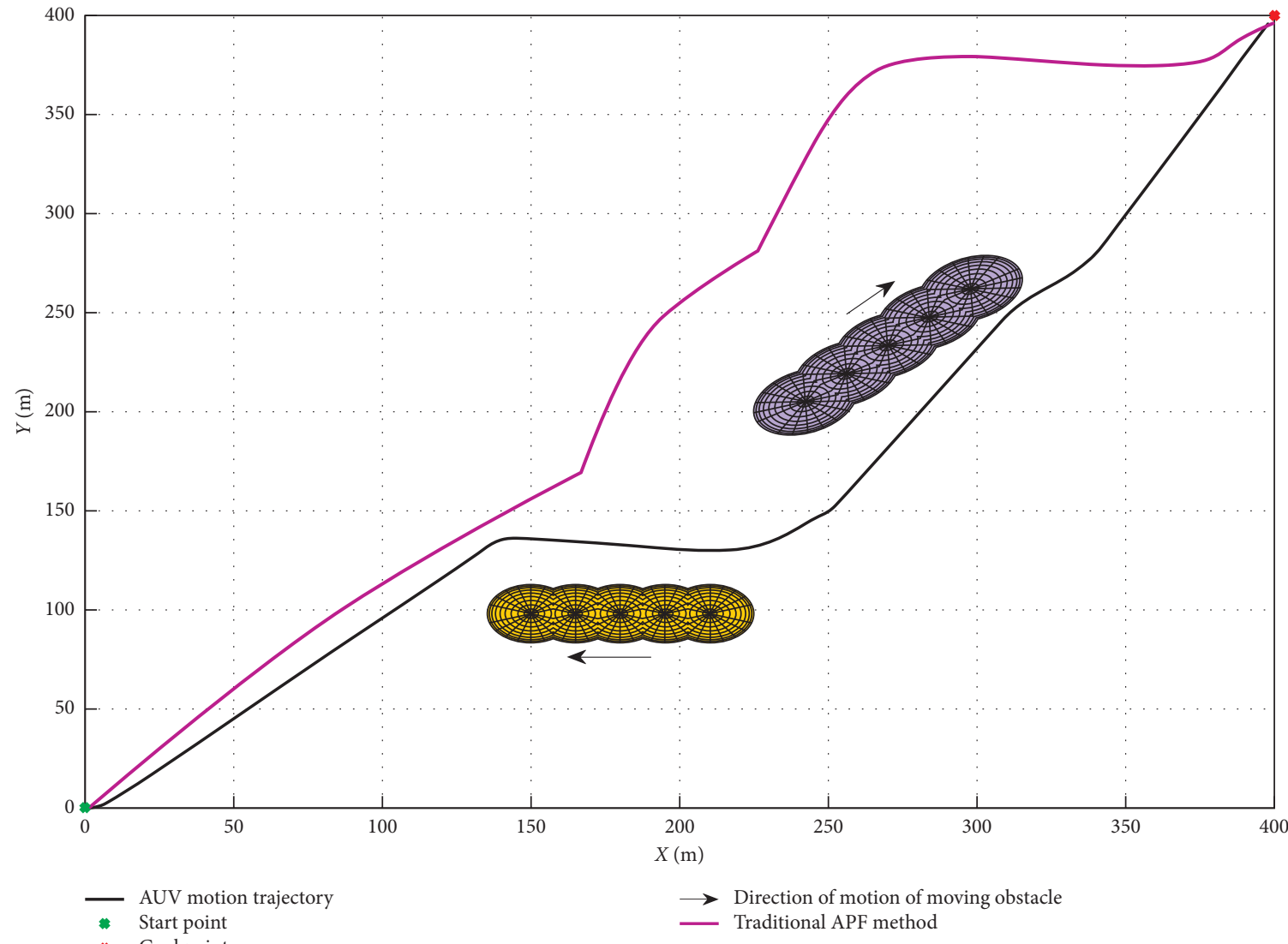

(d)

FIGURE 8: Experimental results of moving obstacles. (a) AUV toward moving obstacle. (b) AUV deviation from moving obstacle. (c) Two moving obstacles. (d) The same direction moving obstacle. 


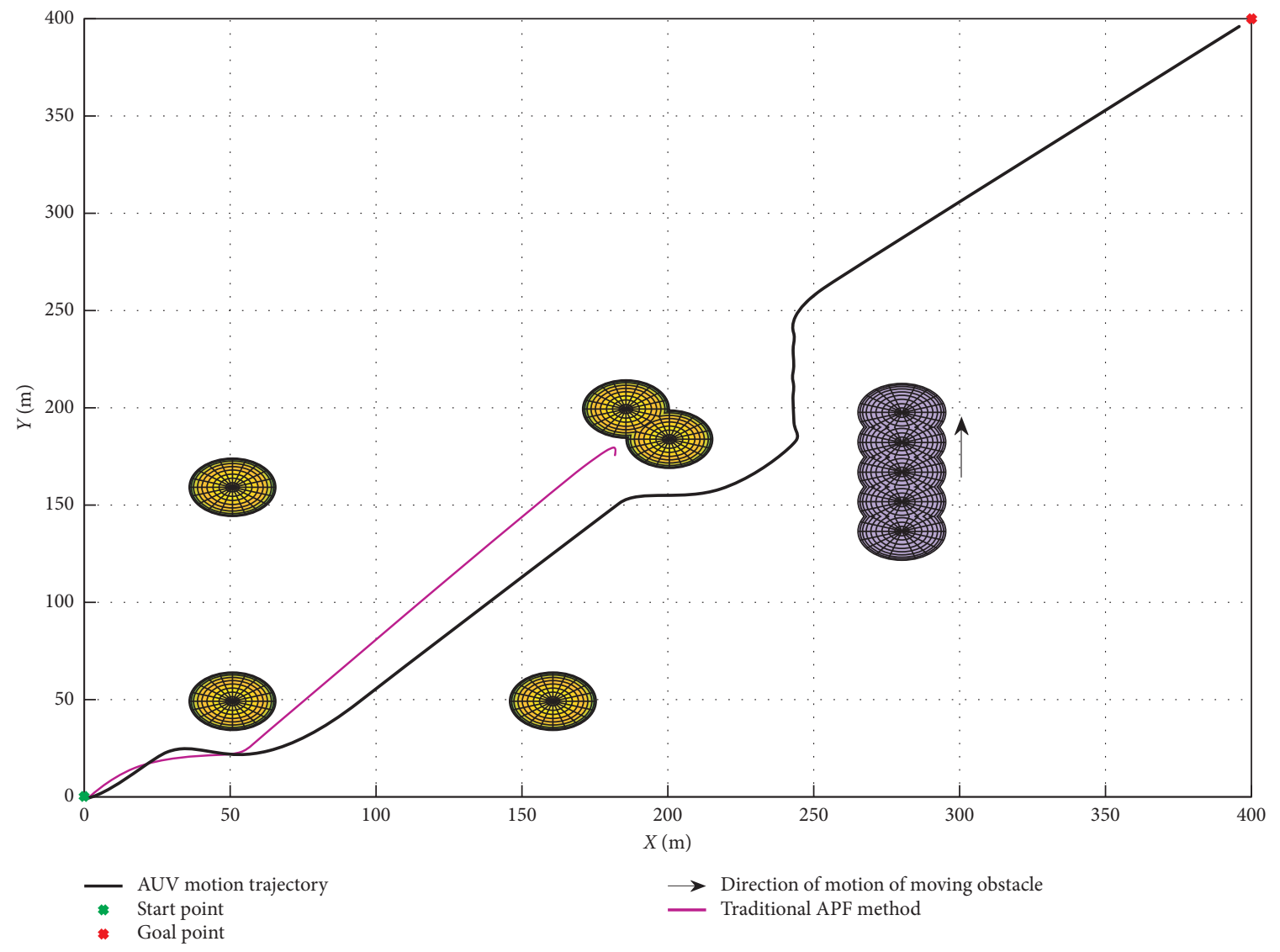

Figure 9: Experimental results of dynamic environment.

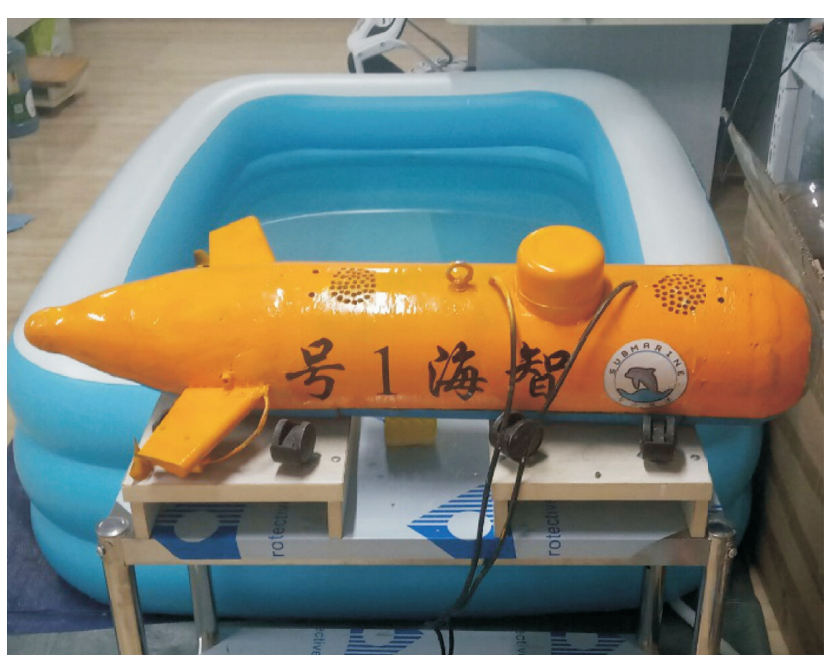

(a)

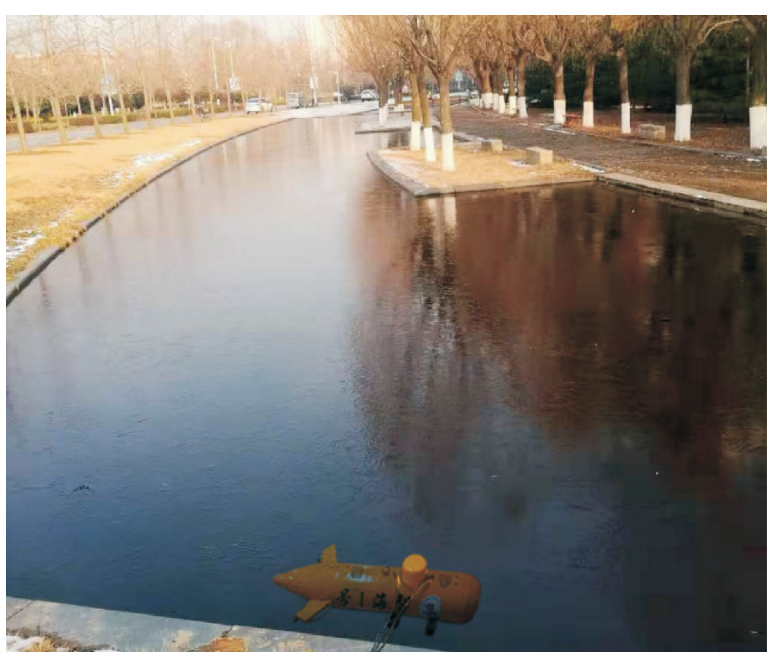

(b)

FIGURE 10: Real environment experimental condition. (a) The IO-I. (b) Experimental environment.

influence range of the obstacle, it goes straight to the goal point without obstacle avoidance. During the whole process of obstacle avoidance, AUV can obtain a smooth motion path.

The relative velocity method can achieve obstacle avoidance for moving obstacles, as shown in Figures 8 and 9. When AUV enters the influence ranges of the moving obstacles, it can determine whether the repulsive potential field function should be modified by judging the relative motion of the obstacle. That can avoid blind obstacle avoidance. If the obstacle speed is similar or faster, AUV can plan a path behind the obstacle. If the obstacle velocity is slower, AUV can plan a path in front of the obstacle. During the real-time obstacle avoidance, the AUV motion path is 


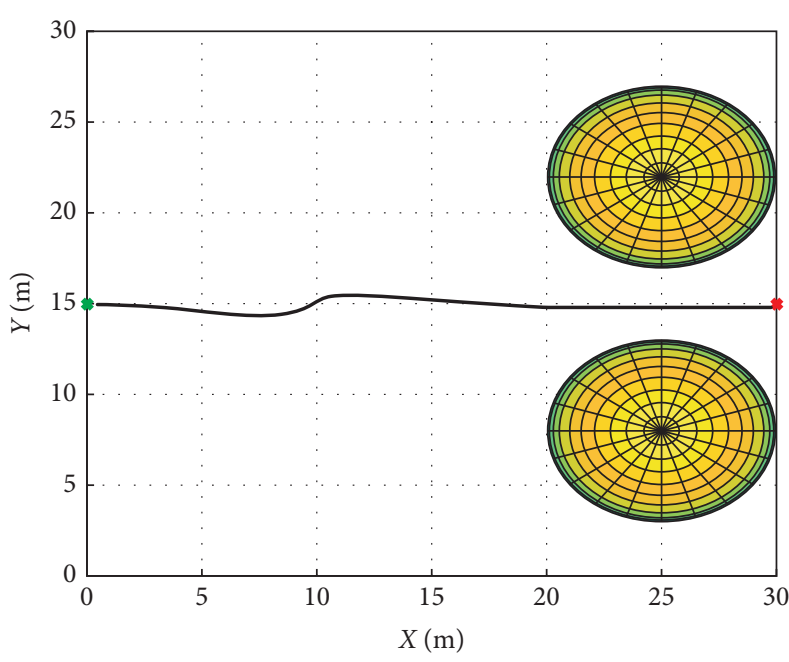

(a)

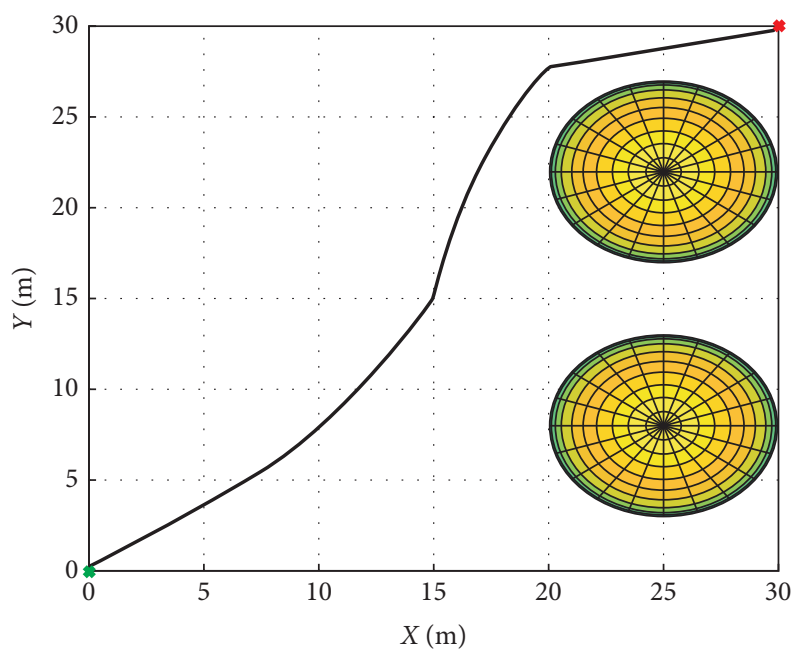

(c)

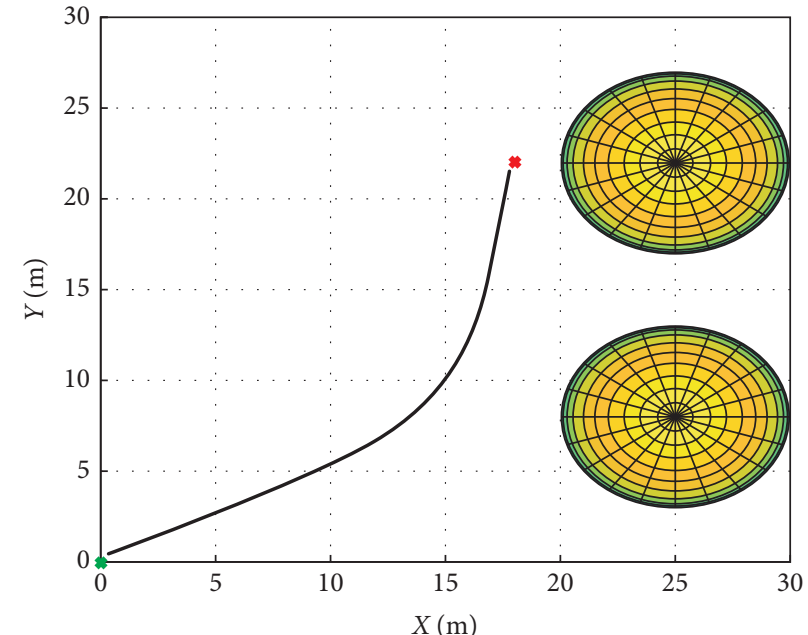

(b)

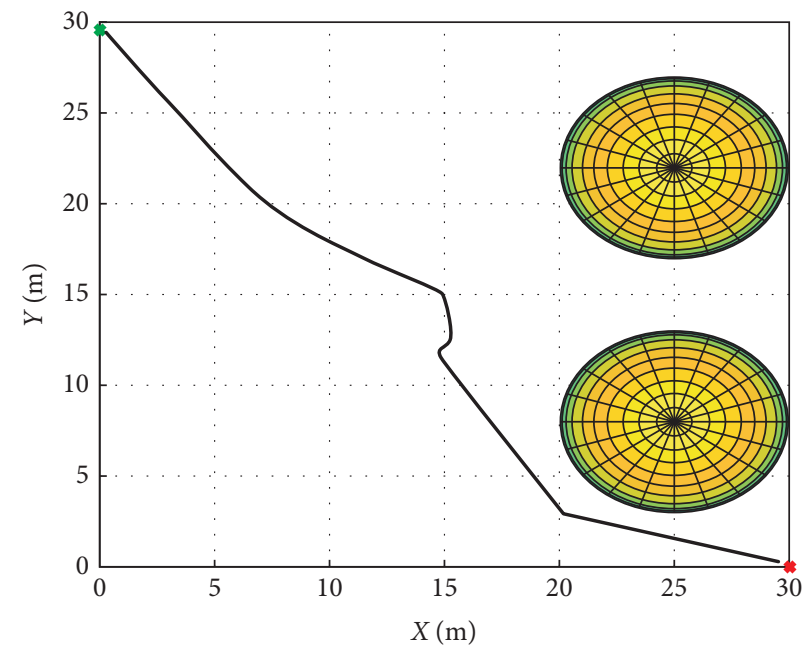

(d)

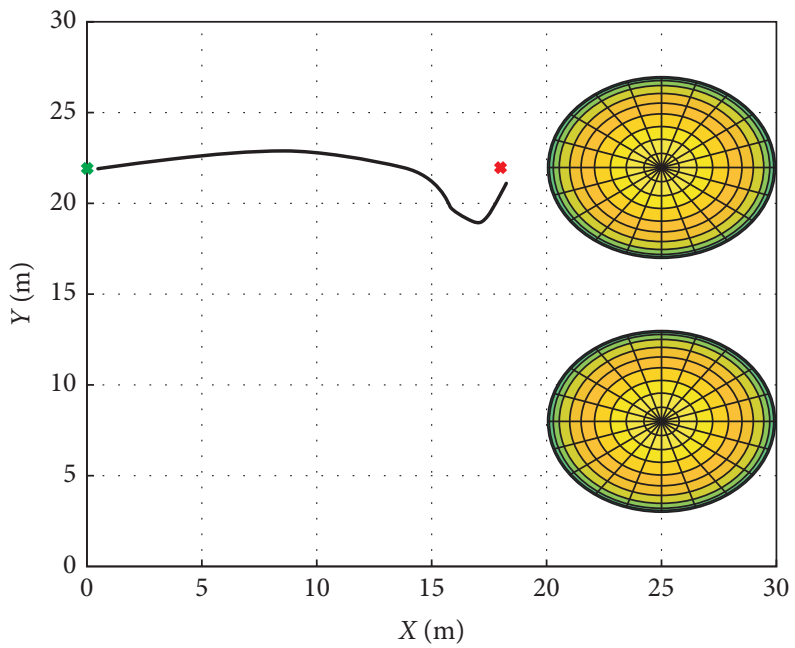

(e)

Figure 11: Experimental results of static obstacles. (a) No local minima situation (obstacles in middle). (b) No local minima situation (the target point in middle). (c) The local minima situation. (d) The local minima situation (exchange start and goal points). (e) The local minima situation (the target point in middle). 


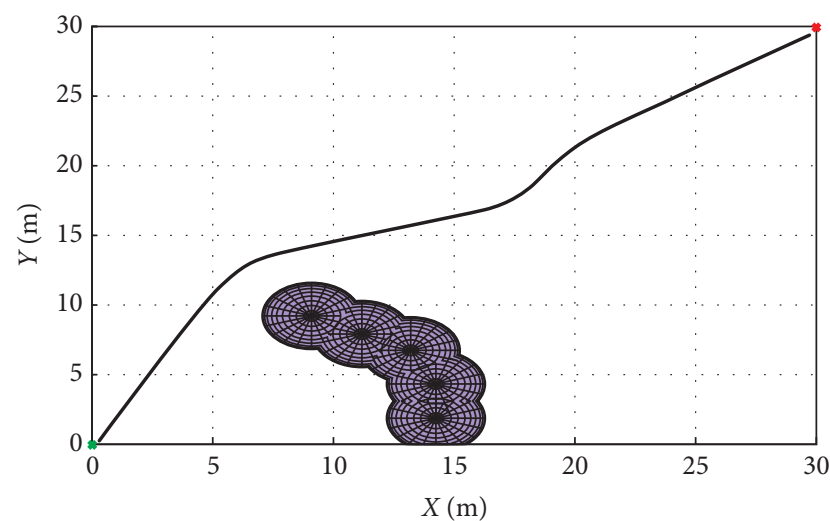

(a)

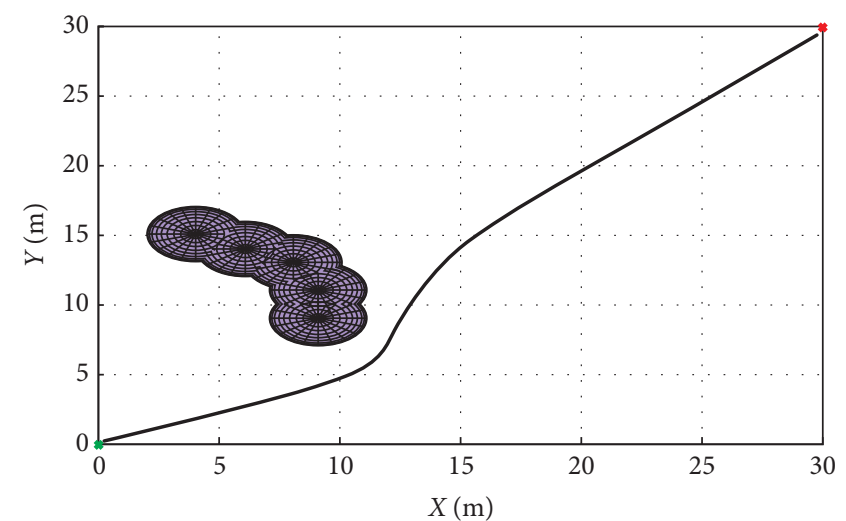

(b)

FIGURE 12: Experimental results of moving obstacles. (a) Slow-moving obstacle. (b) Fast-moving obstacle.

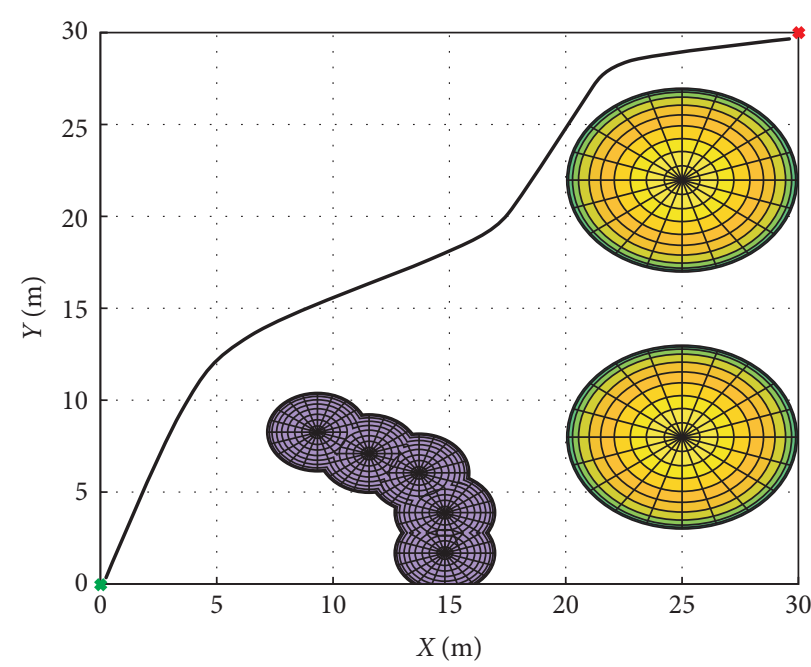

(a)

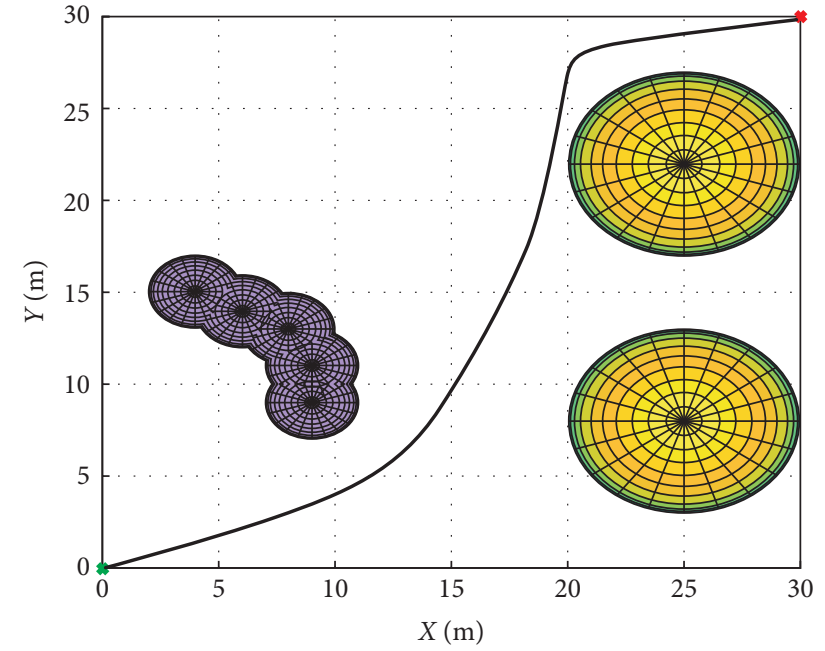

(b)

FIGURE 13: Experimental results of the dynamic environment. (a) Slow-moving obstacle. (b) Fast-moving obstacle.

complete, and there are no oscillations. In summary, the proposed method has promising feasibility and efficiency in the AUV real-time obstacle avoidance. It improves the inherent problems of the traditional APF method while retaining the advantages of real-time nature.

\section{Application and Analysis}

We use an AUV that is named "Intelligence Ocean I" to demonstrate the performance of the proposed method in a practical application. IO-I is an open-frame ARV with dimensions of $1.23 \mathrm{~m} \times 0.24 \mathrm{~m} \times 0.24 \mathrm{~m}$ (length, width, and height). It has two main ducted propellers which are symmetrically arranged on the horizontal stabilizing wings on both sides and two vertical groove thrusters which are, respectively, arranged in the fore and aft along the longitudinal section. The movement of AUV is realized flexibly, including forward, backward, and rotation in the horizontal plane. The motion of the AUV in the depth direction can be realized flexibly also, including up floating, down diving, and pitching motion. The IO-I and experimental environment are shown in Figure 10.

In this section, we show the outcome of path planning, which how the improved APF method and the obstacle avoidance strategy help the AUV avoid the static and moving obstacles. A sphere of acceptance with a radial distance of $0.5 \mathrm{~m}$ is considered at goal position. If the AUV reaches a position within this limit, then the AUV is assumed to have reached the goal position.

5.1. Static Obstacles. We take the convex structure in the experimental environment as static obstacles. The influence range of a single obstacle is set as $d_{0}=5$. The AUV sets off from the initial position at a constant velocity $v=0.5 \mathrm{~m} / \mathrm{s}$.

As shown in Figures 11(a) and 11(b), no local minima situation is encountered. AUV has a smooth planned path along the line between the start point and the goal point. As shown in Figures 11(c) and 11(d), the case of the local minima situation is further classified into two cases to test the performance of the AUV for different starting and goal 
points. AUV has a smooth planned path along the edge of the influence range of obstacles taking advantage of the proposed method. As shown in Figure 11(e), AUV solves the local minima problem using the RHG method and achieves better results.

5.2. Moving Obstacles. The forward speed of the moving obstacle is changed, and the experiment is performed for each condition. The forward speeds are taken as $v_{01}=0.6 \mathrm{~m} / \mathrm{s}$ (fast) and $v_{02}=0.4 \mathrm{~m} / \mathrm{s}$ (slow). The forward speed of the AUV is fixed to a constant value of $v=0.5 \mathrm{~m} / \mathrm{s}$. The proposed method has good performance for fast and slow-moving obstacles according to the experiment results, as shown in Figures 12(a) and 12(b).

For the slow-moving obstacle, AUV plans an obstacle avoidance path in front of the obstacle. For the fast-moving obstacle, AUV plans an obstacle avoidance path behind the obstacle. The relative velocity method makes the planned path more efficient.

5.3. Dynamic Environment. The experimental environment includes two static obstacles and one moving obstacle. AUV makes decisions according to the relative velocity between the moving obstacle and itself and is capable of avoiding the obstacles efficiently for the local minima environment, as shown in Figures 13(a) and 13(b).

\section{Conclusions}

A real-time path planning method based on the modified APF algorithm is presented in this paper. The proposed method can resolve the path planning problem of static and dynamic environments with unknown obstacles (static and moving). AUV can make the decision of obstacle avoidance through apperceiving outside environment and self-state and the motion of obstacles. The distance correction factor is added to the repulsive potential field function based on the traditional APF method to solve the GNRON problem. That can make the AUV reach the goal point rapidly. The RHG method, combined with the APF method, can overcome the local minima problem. When the AUV trapped in a local minimum region, a virtual regular hexagon is constructed. That can guide the AUV moving along the regular hexagon to get rid of the local minima and reaching the goal point. The relative velocity method considers not only the relative distance but also the relative velocity between the AUV and the moving obstacle. It can solve the obstacle avoidance in the presence of moving obstacles. The improvement of the APF algorithm and the feasibility of obstacle avoidance strategy have been evaluated in the simulation environment and the real environment. The experiment results prove that the proposed method can provide feasible and efficient obstacle avoidance paths about both the static and the dynamic obstacles and has good practicability in AUV realtime obstacle avoidance. Besides these, such conclusions can also be drawn as follows: the proposed method inherits the merits of the original APF algorithm and reduces the volume of navigation database and the calculation amount in the process of sailing.

As future work, one work is a further study of safe and real-time navigation. When the obstacle suddenly accelerates or changes its direction of movement, it may cause a collision. Further modifications of the repulsive potential field function or use other methods have to be done for the safety of the AUV navigation. Another future work is continuing to improve the three-dimensional space model that could be beneficial to the forthcoming research and practical applications.

\section{Data Availability}

The data used to support the findings of this study are available from the corresponding author upon request.

\section{Conflicts of Interest}

The authors declare that there are no conflicts of interest regarding the publication of this paper.

\section{Acknowledgments}

The authors wish to acknowledge the financial support of the National Natural Science Foundation of China under Projects 61471224 and 61801270 and Shandong Key Research and Development Program under Grant 2018 GHY115022.

\section{References}

[1] V. A. I. Huvenne, K. Robert, L. Marsh et al., ROVs and AUVs: Submarine Geomorphology, Springer, Berlin, Germany, 2017.

[2] G. X. Wang, G. H. Xu, G. Liu, W. J. Wang, and B. Li, "Fuzzy iterative sliding mode control applied for path following of an autonomous underwater vehicle with large inertia," Mathematical Problems in Engineering, vol. 2019, Article ID 8650243, 14 pages, 2019.

[3] G. X. Yan, G. Pan, and Y. Shi, "Ricochet characteristics of AUVs during small-angle water entry process," Mathematical Problems in Engineering, vol. 2019, Article ID 9518437, 12 pages, 2019.

[4] X. M. Wang and S. G. Liang, "Maneuverability analysis of a novel portable modular AUV," Mathematical Problems in Engineering, vol. 2019, Article ID 1631930, 17 pages, 2019.

[5] H. M. Zhang, L. Yang, and M. L. Li, "Improved ICCP algorithm considering scale error for underwater geomagnetic aided inertial navigation," Mathematical Problems in Engineering, vol. 2019, Article ID 1527940, 9 pages, 2019.

[6] Y. L. Yang, Z. J. Ji, L. Tian, H. Z. Ma, and Q. Y. Qi, "Bipartite consensus of high-order edge dynamics on coopetition multiagent systems," Mathematical Problems in Engineering, vol. 2019, Article ID 1628239, 12 pages, 2019.

[7] X. Cao, C. Y. Sun, and M. Z. Yan, "Optimal disturbances rejection control for autonomous underwater vehicles in shallow water environment," Mathematical Problems in Engineering, vol. 2017, Article ID 8506381, 9 pages, 2017.

[8] P. Ridao, M. Carreras, D. Ribas, P. J. Sanz, and G. Oliver, "Intervention AUVs: the next challenge," Annual Reviews in Control, vol. 40, pp. 227-241, 2015. 
[9] B. W. Wei, W. H. Lyu, X. J. Fan, Y. K. Zhu, and Y. J. Guo, "AUV navigation technology development status and prospect," Journal of Unmanned Undersea Systems, vol. 27, no. 1, pp. 1-9, 2019.

[10] D. Li, P. Wang, and L. Du, "Path planning technologies for autonomous underwater vehicles-a review," IEEE Access, vol. 7, pp. 9745-9768, 2019.

[11] S. M. LaValle, Planning Algorithms, Cambridge University Press, New York,NY, USA, 2006.

[12] J. Han and Y. Seo, "Mobile robot path planning with surrounding point set and path improvement," Applied Soft Computing, vol. 57, pp. 35-47, 2017.

[13] Y. Wang, D. Mulvaney, I. Sillitoe, and E. Swere, "Robot navigation by waypoints," Journal of Intelligent and Robotic Systems, vol. 52, no. 2, pp. 175-207, 2008.

[14] E. Masehian and D. Sedighizadeh, "Classic and heuristic approaches in robot motion planning a chronological review," Proceedings of World Academy of Science: Engineering \& Technology, vol. 23, pp. 101-106, 2007.

[15] P. Bhattacharya and M. Gavrilova, "Roadmap-based path planning-using the voronoi diagram for a clearance-based shortest path," IEEE Robotics \& Automation Magazine, vol. 15, no. 2, pp. 58-66, 2008.

[16] B. Oommen, S. Iyengar, N. Rao, and R. Kashyap, "Robot navigation in unknown terrains using learned visibility graphs. part I: the disjoint convex obstacle case," IEEE Journal on Robotics and Automation, vol. 3, no. 6, pp. 672-681, 1987.

[17] B. Faverjon and P. Tournassoud, "A local based approach for path planning of manipulators with a high number of degrees of freedom," in Proceedings of the IEEE International Conference on Robotics and Automation Proceedings, vol. 4, pp. 1152-1159, Raleigh, NC, USA, April 1987.

[18] A. Blake, R. Cipolla, R. Curwen, Z. Xie, and A. Zisserman, "Visual guidance for robot motion," in Proceedings of the IEE Colloquium on Active and Passive Techniques for 3-D Vision, IEEE, London, UK, February 1991.

[19] C. Cai and S. Ferrari, "Information-driven sensor path planning by approximate cell decomposition," IEEE Transactions on Systems Man and Cybernetics Part B-Cybernetics, vol. 39, no. 3, pp. 672-689, 2009.

[20] X. Yang, M. Moallem, and R. V. Patel, "A layered goal-oriented fuzzy motion planning strategy for mobile robot navigation," IEEE Transactions on Systems, Man and Cybernetics, Part B (Cybernetics), vol. 35, no. 6, pp. 1214-1224, 2005.

[21] Y.-J. Liu, Q. Zeng, S. Tong, C. L. P. Chen, and L. Liu, "Adaptive neural network control for active suspension systems with time-varying vertical displacement and speed constraints," IEEE Transactions on Industrial Electronics, vol. 66, no. 12, pp. 9458-9466, 2019.

[22] M. Z. Zgurovsky and Y. P. Zaychenko, "Fuzzy neural networks in classification problems," The Fundamentals of Computational Intelligence: System Approach, vol. 652, pp. 179-219, 2016.

[23] O. Khatib, "Real-time obstacle avoidance for manipulators and mobile robots," The International Journal of Robotics Research, vol. 5, no. 1, pp. 90-98, 1986.

[24] J. Rintanen, "Planning as satisfiability: heuristics," Artificial Intelligence, vol. 193, no. 24, pp. 45-86, 2012.

[25] P. Hart, N. Nilsson, and B. Raphael, "A formal basis for the heuristic determination of minimum cost paths," IEEE Transactions on Systems Science and Cybernetics, vol. 4, no. 2, pp. 100-107, 1968.

[26] E. W. Dijkstra, "A note on two problems in connexion with graphs," Numerische Mathematik, vol. 1, no. 1, pp. 269-271, 1959.
[27] M. Kurant, A. Markopoulou, and P. Thiran, "Towards unbiased BFS sampling," IEEE Journal on Selected Areas in Communications, vol. 29, no. 9, pp. 1799-1809, 2011.

[28] C.-C. Tsai, H.-C. Huang, and C.-K. Chan, "Parallel elite genetic algorithm and its application to global path planning for autonomous robot navigation," IEEE Transactions on Industrial Electronics, vol. 58, no. 10, pp. 4813-4821, 2011.

[29] M. A. P. Garcia, O. Montiel, O. Castillo, R. Sepúlveda, and P. Melin, "Path planning for autonomous mobile robot navigation with ant colony optimization and fuzzy cost function evaluation," Applied Soft Computing, vol. 9, no. 3, pp. 1102-1110, 2009.

[30] V. Roberge, M. Tarbouchi, and G. Labonte, "Comparison of parallel genetic algorithm and particle swarm optimization for real-time UAV path planning," IEEE Transactions on Industrial Informatics, vol. 9, no. 1, pp. 132-141, 2013.

[31] M. A. Contreras-Cruz, V. Ayala-Ramirez, and U. H. Hernandez-Belmonte, "Mobile robot path planning using artificial bee colony and evolutionary programming," Applied Soft Computing, vol. 30, pp. 319-328, 2015.

[32] L. Amador-Angulo, O. Mendoza, J. R. Castro et al., "Fuzzy sets in dynamic adaptation of parameters of a bee colony optimization for controlling the trajectory of an autonomous mobile robot," Sensors, vol. 16, no. 9, pp. 1-27, 2016.

[33] A. H. Khan, S. Li, X. F. Zhou et al., "Neural \& bio-inspired processing and robot control," Frontiers in Neurorobotics, vol. 12, no. 72, pp. 1-2, 2018.

[34] C. Liu, Y. X. Zhao, F. Gao, and L. Q. Liu, “Three-dimensional path planning method for autonomous underwater vehicle based on modified firefly algorithm," Mathematical Problems in Engineering, vol. 2015, Article ID 561394, 10 pages, 2015.

[35] M. Couillard, J. Fawcett, and M. Davison, "An obstacle recognizing mechanism for autonomous underwater vehicles powered by fuzzy domain ontology and support vector machine," Mathematical Problems in Engineering, vol. 2014, Article ID 676729, 10 pages, 2014.

[36] S. Saravanakumar and T. Asokan, "Multipoint potential field method for path planning of autonomous underwater vehicles in 3D space," Intelligent Service Robotics, vol. 6, no. 4, pp. 211-224, 2013.

[37] F. Matoui, B. Boussaid, and M. N. Abdelkrim, "Distributed path planning of a multi-robot system based on the neighborhood artificial potential field approach," Simulation, vol. 95, no. 7, pp. 637-657, 2019.

[38] T. Weerakoon, K. Ishii, and A. A. F. Nassiraei, "An artificial potential field based mobile robot navigation method to prevent from deadlock," Journal of Artificial Intelligence and Soft Computing Research, vol. 5, no. 3, pp. 189-203, 2015.

[39] J. B. Sun, G. L. Liu, G. H. Tian, and J. H. Zhang, "Smart obstacle avoidance using a danger index for a dynamic environment," Applied Sciences, vol. 9, no. 8, p. 1589, Apr. 2019.

[40] X. X. Liang, C. Y. Liu, X. L. Song, and Y. K. Zhang, "Research on improved artificial potential field approach in local path planning for mobile robot," Computer Simulation, vol. 35, no. 4, pp. 291-361, 2018.

[41] X. Yang, W. Yang, H. J. Zhang et al., "A new method for robot path planning based artificial potential field," in Proceedings of the IEEE 11th Conference on Industrial Electronics and Applications, Hefei, China, June 2016.

[42] G. Li, A. Yamashita, H. Asama, and Y. Tamura, "An efficient improved artificial potential field based regression search method for robot path planning," in Proceedings of the IEEE 12th International Conference on Mechatronics and Automation, Chengdu, China, December 2012. 
[43] S. S. Ge and Y. J. Cui, "Dynamic motion planning for mobile robots using potential field method," Autonomous Robots, vol. 13, no. 3, pp. 207-222, 2002.

[44] O. Montiel, R. Sepúlveda, and U. Orozco-Rosas, "Optimal path planning generation for mobile robots using parallel evolutionary artificial potential field," Journal of Intelligent \& Robotic Systems, vol. 79, no. 2, pp. 237-257, 2015.

[45] D. Q. Zhu, C. L. Cheng, and B. Sun, “An integrated AUV path planning algorithm with ocean current and dynamic obstacles," International Journal of Robotics \& Automation, vol. 31, no. 5, pp. 382-389, 2016.

[46] C. L. Cheng, D. Q. Zhu, B. Sun et al., "Path planning for autonomous underwater vehicle based on artificial potential field and velocity synthesis," in Proceedings of the Canadian Conference on Electrical and Computer Engineering, pp. 717-721, Halifax, Canada, May 2015. 